\title{
Current trends and geographical differences in therapeutic profile and outcomes of COVID-19 among pregnant women - a systematic review and meta-analysis
}

Pallavi Dubey', Bhaskar Thakur ${ }^{2}$, Sireesha Reddy ${ }^{1}$, Carla A. Martinez ${ }^{1}$, Md Nurunnabi $^{3}$, Sharron L. Manuel' Sadhana Chheda ${ }^{4}$, Christina Bracamontes ${ }^{1}$ and Alok K. Dwivedi ${ }^{2,5^{*}}$ (i)

\begin{abstract}
Background: Coronavirus disease (COVID-19) has been associated with adverse pregnancy outcomes. Due to the lack of effective treatments for COVID-19, it becomes imperative to assess the geographical differences and trends in the current clinical care and outcomes of COVID-19 in pregnant women.

Methods: A PubMed search was performed to screen articles reporting therapeutics and outcomes of confirmed COVID-19 in pregnant women prior to August 27, 2020. We performed searches, quality assessments of eligible studies, extracted and reported data according to PRISMA guidelines. Meta-analyses and cumulative meta-analyses of proportions were performed for estimating each outcome and their pattern over time respectively.
\end{abstract}

Results: One thousand two hundred thirty nine pregnant women with COVID-19 from 66 studies were analyzed. In case series analysis reflecting average-risk patients, the proportion of oxygen support, antibiotics, antivirals, and plasma therapy administration except for hydroxychloroquine was substantially higher in Asian studies $(55,78,80,6$, and $0 \%)$ compared to the US $(7,1,12,0$, and $7 \%)$ or European $(33,12,14,1$, and $26 \%)$ studies, respectively. The highest preterm birth and the average length of hospital stay (35\%, 11.9 days) were estimated in Asian studies compared to the US studies (13\%, 9.4 days) and European studies (29\%, 7.3 days), respectively. Even in case reports reflecting severe cases, the use of antivirals and antibiotics was higher in Asian studies compared to the US, Latin American, and European studies. A significant decline in the use of most therapeutics along with adverse outcomes of COVID-19 in pregnant women was observed.

(Continued on next page)

\footnotetext{
* Correspondence: alok.dwivedi@ttuhsc.edu

2Division of Biostatistics \& Epidemiology, Department of Molecular and

Translational Medicine, Paul L. Foster School of Medicine, Texas Tech

University Health Sciences Center El Paso, El Paso, TX, USA

${ }^{5}$ Biostatistics and Epidemiology Consulting Lab, Texas Tech University Health

Sciences Center El Paso, El Paso, TX, USA

Full list of author information is available at the end of the article
}

(c) The Author(s). 2021 Open Access This article is licensed under a Creative Commons Attribution 4.0 International License, which permits use, sharing, adaptation, distribution and reproduction in any medium or format, as long as you give appropriate credit to the original author(s) and the source, provide a link to the Creative Commons licence, and indicate if changes were made. The images or other third party material in this article are included in the article's Creative Commons licence, unless indicated otherwise in a credit line to the material. If material is not included in the article's Creative Commons licence and your intended use is not permitted by statutory regulation or exceeds the permitted use, you will need to obtain permission directly from the copyright holder. To view a copy of this licence, visit http://creativecommons.org/licenses/by/4.0/ The Creative Commons Public Domain Dedication waiver (http://creativecommons.org/publicdomain/zero/1.0/) applies to the data made available in this article, unless otherwise stated in a credit line to the data. 
(Continued from previous page)

Conclusions: Geographical differences in therapeutic practice of COVID-19 were observed with differential rates of maternal and clinical outcomes. Minimizing the use of some therapeutics particularly antibiotics, antivirals, oxygen therapy, immunosuppressants, and hydroxychloroquine by risk stratification and careful consideration may further improve maternal and clinical outcomes.

Keywords: Treatment, Hydroxychloroquine, Antivirals, Preterm birth, Antibiotics, Mechanical ventilation, ICU admission, Maternal death, Adverse pregnancy outcomes, Meta-analysis, Cesarean section

\section{Background}

An estimated 27 million people worldwide have been infected with the coronavirus disease 2019 till October $2020[1,2]$. SARS-CoV-2 infection seems less virulent than the Severe Acute Respiratory Syndrome (SARS) and Middle East Respiratory Syndrome (MERS) in terms of morbidity and mortality [3, 4]. We and others have observed high rates of adverse pregnancy outcomes including preterm birth among COVID-19 women [5]. There are no established therapies for COVID-19 particularly in pregnant women. Hence, it becomes imperative to provide observational evidence of the current therapeutic practice of COVID-19 in clinical care for the management of pregnant women.

The most common therapeutics for managing COVID-19 in pregnant women were antibiotics, antivirals, and oxygen supports. Intensive care and mechanical ventilation (MV) is needed to deal with disease severity [6]. Lopinavir / Ritonavir, a HIV-1 protease inhibitor [7] has been used as a treatment option for COVID-19 as an antiviral, however, there is no clear benefit observed in the treatment of COVID-19 [8]. In contrast, remdesivir [9] and dexamethasone [10] are considered acceptable treatments with evidence for hospitalized and severe COVID-19 patients. Tocilizumab as an IL-6 inhibitor has been used for treating severe and critical COVID-19 cases in the US [11]. Although hydroxychloroquine (HCQ) initially received emergency use authorization and then revoked by the Food and Drug Administration [12], HCQ has been used for treating COVID-19 patients [13]. The use of antibiotics has also been observed in COVID-19 cases without any bacterial infection [14]. Convalescent plasma therapy and anticoagulants have been recommended for the treatment of hospitalized COVID-19 patients and critical patients [15, 16]. In addition, the potential benefits of zinc/magnesium have been demonstrated especially in elderly or immunocompromised patients and thus these treatments have been used for managing COVID-19 as well [17].

Information on maternal and pregnancy outcomes after treatment for COVID-19 has been limited [18]. During ongoing COVID-19 pandemic, understanding the chronological pattern of therapeutic use as per their benefits and risks corresponding to the pattern in maternal and clinical outcomes may help health care management to make an appropriate decision in the increase or decrease use of specific therapy or medication. In our previous study [19], we observed the geographical differences in pregnancy outcomes showing more prevalent adverse pregnancy outcomes in China compared to the US and Europe. Although pregnant women have an increased risk of hospitalization and intensive care unit (ICU) admission [6], it is still unknown if there are any geographical differences persist in COVID-19 outcomes as well.

\section{Methods}

\section{Search methods}

We followed the Preferred Reporting Items for Systematic Reviews and Meta-analyses (PRISMA) [20] and the Meta-analysis of Observational Studies in Epidemiology (MOOSE) [21] guidelines [22]. A comprehensive search was performed on PubMed for screening any studies reporting data on COVID-19 in pregnant women prior to August 27th, 2020. We used the combinations of search terms "COVID-19 OR SARS-CoV-2 OR Coronavirus" AND "Pregnancy OR Pregnant" to screen articles. References and review studies were utilized to screen any eligible studies excluded from the initial search (Fig. 1). The two authors (PD and SR) independently reviewed all studies for their eligibility. An extensive review of all articles was conducted by two authors (BT and PD) to exclude any duplicated articles based on the recruitment period, location, or authors of studies.

\section{Inclusion and exclusion criteria}

Any study reporting treatments for managing SARSCoV-2 infection in pregnant women in outpatients setting or during hospitalization was considered eligible for this systematic review and meta-analysis study. All pregnant patients with a confirmed COVID-19 infection by quantitative real-time polymerase chain reaction with or without maternal or clinical outcomes were only included in this study. Unpublished studies/reports and studies that could not be translated into the English language or indicating duplicative data were excluded from the analysis. 


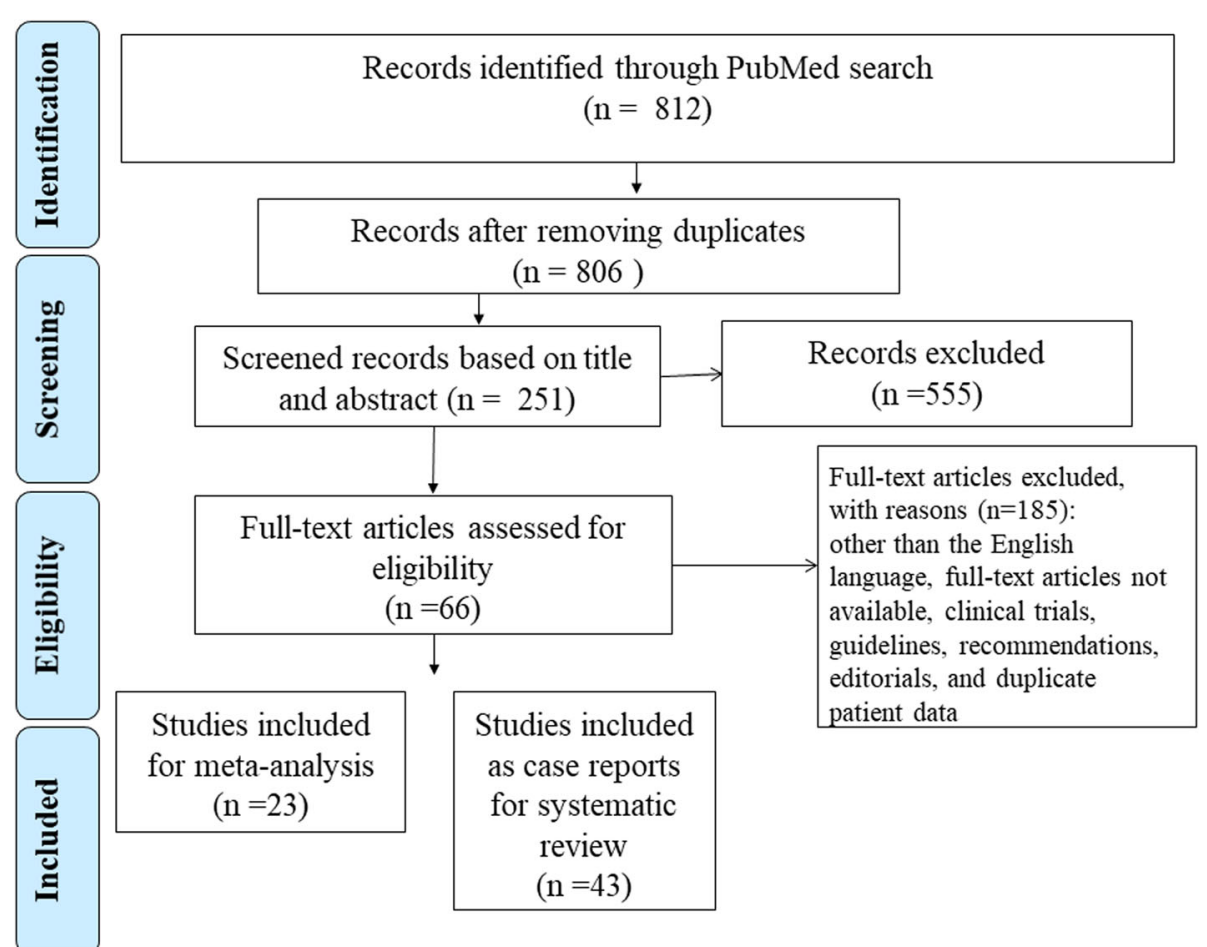

Fig. 1 PRISMA flowchart for study selection

\section{Study endpoints}

We extracted data on a) treatment profile including oxygen support, corticosteroids, immunosuppressants, $\mathrm{HCQ}$, antivirals, zinc/magnesium, anticoagulants, antibiotics, plasma therapy, and mechanical ventilation b) maternal and neonatal outcomes including pregancy status (delivered or still pregnant), number of neonates, delivery type (cesarean section or vaginal), maternal death, fetal demise, neonatal death, c) clinical outcomes of COVID-19 including intensive care unit (ICU) admission and hospital length of stay (HLOS) d) maternal characteristics including age range, gestational age, comorbidities (diabetes-DM, hypertension-HTN, asthma, obesity), symptomatic or asymptomatic status of COVID-19. The primary outcomes in the study were therapeutics for managing COVID-19 in pregnant women, preterm birth, ICU admission, and HLOS. Maternal death and fetal demise rates were included as secondary outcomes. Studies with more than 5 cases were considered as case series studies otherwise case reports. The location of the study was classified into Asia, United States of America (USA), Europe, and Latin America. However, data from Latin American countries was only available in case reports studies.

\section{Assessments of the risk of bias}

We conducted the quality assessment of all eligible case series studies using the quality assessment tool provided by the National Heart, Lung, and Blood Institute, Research Triangle Institute International. In addition, we performed an assessment of publication bias using funnel plots and Egger's test for primary outcomes only.

\section{Statistical analysis}

For case series studies, the random effects meta-analysis of proportions was carried out for each binary outcome using the Dersimonian and Laird (D-L) method with the Freeman-Tukey double arcsine transformation to obtain the estimates. The results of the D-L method were summarized using a pooled proportion with a 95\% confidence interval (CI). The $\mathrm{I}^{2}$ statistic was used to assess the heterogeneity across effect sizes. The average HLOS was pooled across the studies using the weighted generalized linear model (GLM) with the Gaussian family and identity link function. The studies were weighted according to the sample size of each study, larger sample size studies received a higher weight relative to smaller sample size studies. Cumulative meta-analysis for each outcome was performed by the month of each publication to obtain a pattern in outcome over the pandemic period. The subgroup analyses for ICU, HLOS, and preterm birth were also conducted using the D-L random effects models or weighted GLM according to low or high use of each of the therapeutics, comorbidities, cesarean section rate, and symptomatic clinical presentation. For the systematic review of case reports, we 
performed individual level descriptive data analysis and applied a Fisher's exact test or an unpaired t-test or a Pearson's correlation analysis. All the statistical computations were carried out by the STATA statistical software (version 15.1).

\section{Results}

Eight hundred twelve studies were screened and two hundred fifty-one were evaluated for data extraction. Of 251 studies, 66 fulfilled the eligibility criteria yielding 1239 COVID-19 cases for data analysis. Twenty-three studies were included in meta-analysis while 43 studies reporting 64 cases were included in the systematic review and qualified for descriptive data analysis (Table 1). Qualitative assessment and bias evaluation were performed on all the 23 studies eligible for the metaanalysis. Most of the studies included in the metaanalysis were of fair/good quality (Supplementary Table 1) without any sign of publication bias (Supplementary Table 2, Supplementary Fig. 1 A and B).

Maternal characteristics, treatment profile, and outcomes In the analysis of the 1239 pregnant women, 944 subjects had a delivery while 295 women were still pregnant at the end of the study. In case series, the average maternal age was 30.6 years (mid-range: $25-37$ ) with average gestational age of 31.3 (mid-range: 22-43) weeks. The average BMI was 29.4 (mid-range: 25.3-35.8) $\mathrm{kg} / \mathrm{m}^{2}$. Overall $9 \%$ of patients had obesity and $4 \%$ had DM, HTN, or asthma. Most patients presented with a symptom of COVID-19 (89\%). The most common therapeutic was antibiotics (36\%) followed by oxygen support (33\%), antivirals (33\%), HCQ (10\%), anticoagulants (3\%), and plasma support (2\%). The proportion of mechanical ventilation was estimated to be $3 \%$ with a $6 \%$ ICU admission rate (95\%CI: 2, 10\%). The average HLOS was 8.5 days (95\%CI: 5.96, 10.97). The cesarean section and preterm birth rates were estimated to be 62 and $26 \%$ respectively. Fetal demise was less than 1\% with 20 maternal deaths (Table 2).

In case reports, the average maternal age, gestational age, and average BMI were 31.1 (mid-range: 22-44) years, 32.7 (mid-range: 21-40) weeks, and 35.8 (midrange: $25-57) \mathrm{kg} / \mathrm{m}^{2}$ respectively. The most common comorbidities were obesity (30\%) and DM (23\%) followed by HTN and asthma (9\%). The majority of patients had a symptomatic presentation (89\%). The proportion of antibiotics, oxygen support, antivirals, steroids, $\mathrm{HCQ}$, zinc/magnesium, plasma therapy, anticoagulants, and immunosuppressants was $64,53,45,44$, $26,21,14,12$, and $10 \%$ respectively. Over one-third of the patients required mechanical ventilation support (35\%) with 43\% ICU admission rate (95\% CI: 31, 57\%). The average HLOS was estimated to be 14.6 days
(95\%CI: 12.0-17.2). The rate of cesarean section, preterm birth, fetal demise, and maternal death was 69,50 , 8 , and $5 \%$ respectively. In addition, $2 \%$ neonatal deaths were recorded (Table 2).

\section{Meta-analysis of maternal characteristics, treatment profile, and outcomes by geographic location}

The average age of patients was highest in Europe (32.1 years) followed by the US (30.8 years) and Asia (28.4 years). However, the average gestational age at detection was lowest in European studies (29.3 weeks) and the US studies (31.9 weeks) compared to Asian studies (34.8 weeks). Most of the pregnant patients were symptomatic in Asian (100\%) and European (92\%) studies, while almost half of the patients were asymptomatic in the US studies. Among comorbidities, obesity was most commonly reported in the US (20\%) and European studies (11\%). Among therapeutics, antibiotics were most commonly observed in Asia (78\%) while oxygen support (33\%) and HCQ (26\%) were the most common therapeutics in Europe. Although with low proportions, antibiotics (12\%), HCQ (7\%), and oxygen support (7\%) were relatively more used in the US patients compared to other treatments. The least use of any therapeutics except for HCQ was obtained in the US studies relative to other countries. European studies had higher proportions of HCQ and corticosteroid use than the US and Asia. The proportions of mechanical ventilation support (2, 4, and 6\%) and ICU admission (6, 5, and 7\%) were found to be similar across the US, Asia, and Europe, respectively. However, the average HLOS was highest in Asian studies (11.8 days) and least in European studies (7.34 days). The preterm birth rate was relatively higher in Asian (35\%) and European studies (29\%) compared to the US studies (13\%). The cesarean section rate was similar between US (46\%) and European studies (53\%) but lower than Asian studies (80\%) (Table 3).

\section{Cumulative meta-analysis of treatments and outcomes over time}

In general, a decline in the use of most therapeutics was observed over time except for HCQ and corticosteroid use. A slight increase in the use of HCQ and corticosteroids has started to begin since early May, 2020 (Supplementary Fig. 2). Like the decline in therapeutic use, the reduction in all outcomes (ICU admission, HLOS, and preterm birth and cesarean section rates) over time was also observed (Figs. 2 and 3). Among therapeutics, the greatest decline from May to August was 24\% in antibiotics, $23 \%$ in antivirals, $11 \%$ in oxygen support (Fig. 4) while no change or slight gain in other therapeutics was observed. Only a $13 \%$ change in cesarean section and $2 \%$ change in preterm birth with 2 days reduction in HLOS were estimated between May to August without any 
Table 1 Study characteristics

\begin{tabular}{|c|c|c|c|c|c|c|c|c|c|c|c|c|c|c|}
\hline PMID & Author & Month & Country & $\mathbf{N}$ & Age(y) & GW & $\begin{array}{l}\mathrm{BMI} \\
\left(\mathrm{kg} / \mathrm{m}^{2}\right)\end{array}$ & $\mathrm{DM}$ & HT & Asthma & Obesity & ICU & $\begin{array}{l}\text { HLOS } \\
\text { (days) }\end{array}$ & Preterm \\
\hline \multicolumn{15}{|l|}{ Case series } \\
\hline 32151335 & Chen et al. [23] & March & China & 9 & 33 & 37.1 & NA & NA & NA & NA & NA & 0 & NA & 4 \\
\hline 32186894 & Liu et al. [24] & March & China & 15 & 31.5 & 25 & NA & 1 & 0 & 0 & 0 & NA & NA & 3 \\
\hline 32249918 & Li et al. [25] & March & China & 16 & 31.5 & 36.5 & NA & 3 & 3 & 0 & 0 & 0 & 14.5 & 13 \\
\hline 32285380 & Liu et al. [26] & March & China & 19 & 31 & 38 & NA & NA & NA & NA & NA & 0 & NA & 2 \\
\hline 32360108 & Hantoushzadeh et al. [27] & April & Iran & 9 & 37 & 43 & 27 & 1 & 0 & 0 & 1 & 9 & NA & 6 \\
\hline 32428964 & London et al. [28] & April & USA & 68 & 29.6 & NA & 30.25 & 7 & 2 & 2 & & 1 & 14.5 & 9 \\
\hline 32696241 & Chen et al. [29] & April & China & 21 & 29 & NA & NA & 3 & 0 & 0 & 0 & 0 & & \\
\hline 32433453 & Savasi et al. [30] & May & Italy & 77 & 31.5 & 23 & 35.8 & NA & NA & NA & NA & 14 & NA & 12 \\
\hline 32438521 & Zeng et al. [31] & May & China & 16 & 32.5 & 37 & NA & NA & NA & NA & NA & 0 & NA & 3 \\
\hline 32439389 & Lokken et al. [32] & May & USA & 46 & 30 & 27.4 & NA & 3 & 2 & 4 & 15 & 1 & 4.5 & 1 \\
\hline 32632417 & San-Juan et al. [33] & May & Spain & 32 & 32 & 29 & NA & 2 & 0 & 4 & 1 & 2 & 7.5 & NA \\
\hline 32641013 & Zhang et al. [34] & May & China & 18 & 29 & 38 & NA & NA & NA & NA & NA & 0 & NA & 3 \\
\hline 32553908 & Sentilhes et al. [35] & June & France & 54 & 30.6 & 30.4 & 25.3 & 0 & 1 & 5 & 4 & 4 & 4 & NA \\
\hline 32553910 & Blitz et al. [36] & June & USA & 43 & 29.5 & 35 & 30.9 & 1 & 0 & 2 & 5 & 13 & 16 & 1 \\
\hline 32633022 & Prabhu et al. [37] & July & USA & 70 & 31.6 & 38.6 & 31 & 6 & 11 & 6 & 12 & 1 & 3.6 & 11 \\
\hline 32633712 & Vivanti et al. [38] & July & France & 100 & 32.8 & 27 & 27.05 & 7 & 6 & 9 & 10 & 10 & 9.1 & 20 \\
\hline 32649784 & Gabriel et al. [39] & July & Spain & 42 & 33.6 & 37.6 & NA & NA & NA & NA & NA & 3 & NA & 9 \\
\hline 32682342 & Sahin et al. [40] & July & Turkey & 29 & 28.5 & 22 & 28 & 0 & 0 & 1 & 5 & 0 & 11 & 2 \\
\hline 32689846 & Barbero et al. [41] & July & Spain & 91 & 33.25 & 28 & 26.2 & 3 & 3 & 5 & 20 & 4 & NA & 8 \\
\hline 32701761 & Emeruwa et al. [42] & July & USA & 100 & 32 & 28 & 30.7 & 3 & 17 & 12 & NA & NA & NA & 13 \\
\hline 32743014 & Xu et al. [43] & July & China & 34 & 32.5 & 28 & NA & 2 & 1 & 0 & 0 & 1 & 10.6 & 5 \\
\hline 32776309 & Oncel et al. [44] & August & Turkey & 125 & NA & 34.5 & NA & 7 & 8 & 0 & NA & 8 & 6.5 & 33 \\
\hline 32760169 & Nayak et al. [45] & August & India & 141 & 25 & 35.5 & NA & 4 & 7 & 2 & NA & NA & NA & NA \\
\hline Summary & & & & 1175 & 30.60 & 31.30 & 29.40 & 53 & 61 & 52 & 73 & 71 & 8.47 & 158 \\
\hline \multicolumn{15}{|l|}{ Case report } \\
\hline 32119083 & Wang et al. [46] & February & China & 1 & 28 & 30 & NA & NA & NA & NA & NA & 0 & 19 & 1 \\
\hline 32134381 & Li et al. [47] & March & China & 1 & 30 & 35 & NA & 0 & 0 & 0 & 0 & 0 & 15 & 1 \\
\hline 32161941 & Wang et al. [48] & March & China & 1 & 34 & 40 & NA & 0 & 0 & 0 & 0 & 0 & 18 & 0 \\
\hline 32182347 & Fan et al. [49] & March & China & 2 & 31.5 & 36.2 & NA & NA & NA & NA & NA & 0 & 20 & 1 \\
\hline 32222119 & Chen et al. [50] & March & China & 5 & 28 & 39.5 & NA & NA & NA & NA & NA & 0 & NA & 0 \\
\hline 32229802 & Lee et al. [51] & March & Korea & 1 & 35 & 37 & NA & NA & NA & NA & NA & 1 & 1 & 0 \\
\hline 32249471 & Kalafat et al. [52] & March & Turkey & 1 & 32 & 35 & NA & 0 & 0 & 0 & 0 & 1 & 9 & 1 \\
\hline 32249924 & Gidlöf et al. [53] & March & Sweden & 1 & 34 & 36 & 38 & 1 & 0 & 0 & 1 & 0 & NA & 2 \\
\hline 32279693 & Khan et al. [54] & March & China & 3 & 30 & 36.5 & NA & NA & NA & NA & NA & 0 & NA & 1 \\
\hline 32237670 & lqbal et al. [55] & April & USA & 1 & 34 & 39 & NA & NA & NA & NA & NA & 0 & 6 & 0 \\
\hline 32305046 & Alzamora et al. [56] & April & Peru & 1 & 41 & 33 & 35 & 0 & 0 & 0 & 2 & 0 & 3 & 1 \\
\hline 32305459 & Peng et al. [57] & April & China & 1 & 25 & 35 & NA & NA & NA & NA & NA & 0 & 14 & 1 \\
\hline 32313302 & Romero et al. [58] & April & Spain & 1 & 44 & 29 & NA & 0 & 0 & 0 & 0 & 1 & NA & NA \\
\hline 32330313 & Lu et al. [59] & April & China & 1 & 22 & 38 & NA & 0 & 0 & 0 & 0 & 0 & 16 & 0 \\
\hline 32330970 & Browne et al. [60] & April & USA & 1 & 33 & 23 & NA & 0 & 0 & 1 & 0 & 0 & 4 & 0 \\
\hline 32384385 & Blauvelt et al. [61] & April & USA & 1 & 34 & 28 & NA & 1 & 0 & 1 & 1 & 1 & 16 & 1 \\
\hline 32509416 & Silverstein et al. [62] & April & USA & 2 & 25.5 & 35 & 40 & 0 & 0 & 0 & 1 & 2 & 19 & 2 \\
\hline 32523874 & AlZagha et al. [63] & April & Jordan & 1 & 30 & 36 & NA & 0 & 0 & 0 & 0 & 0 & 11 & 1 \\
\hline 32369616 & Li et al. [64] & May & China & 1 & 31 & 35 & NA & 0 & 0 & 0 & 0 & 1 & 45 & 1 \\
\hline
\end{tabular}


Table 1 Study characteristics (Continued)

\begin{tabular}{|c|c|c|c|c|c|c|c|c|c|c|c|c|c|c|}
\hline PMID & Author & Month & Country & $\mathrm{N}$ & Age(y) & GW & $\begin{array}{l}\text { BMI } \\
\left(\mathrm{kg} / \mathrm{m}^{2}\right)\end{array}$ & $\mathrm{DM}$ & HT & Asthma & Obesity & ICU & $\begin{array}{l}\text { HLOS } \\
\text { (days) }\end{array}$ & Preterm \\
\hline 32382516 & Hong et al. [65] & May & USA & 1 & 36 & 23 & 41.53 & 0 & 0 & 0 & 1 & 1 & 11 & 0 \\
\hline 32405454 & Taghizadieh et al. [66] & May & Iran & 1 & 33 & 34 & NA & 0 & 0 & 0 & 0 & 1 & NA & 1 \\
\hline 32425297 & Cooke et al. [67] & May & UK & 2 & 33.5 & 28 & 42 & 1 & 0 & 0 & 1 & 0 & 7 & 2 \\
\hline 32426242 & Mehta et al. [68] & May & USA & 1 & 39 & 27 & NA & 0 & 0 & 0 & 0 & 1 & 11 & 2 \\
\hline 32426243 & Anderson et al. [8] & May & USA & 1 & 35 & 22 & NA & 1 & 0 & 1 & 1 & 0 & 14 & 0 \\
\hline 32428290 & Yu et al. [69] & May & China & 1 & 35 & 34 & NA & 0 & 0 & 0 & 0 & 1 & 12 & 1 \\
\hline 32505514 & Fontanella et al. [70] & May & & 2 & 34 & 35.5 & 43 & 1 & 0 & 0 & 0 & 0 & 3.5 & 0 \\
\hline 32606133 & Grimminck et al. [71] & June & Netherlands & 1 & 31 & 38 & NA & 0 & 1 & 0 & 0 & 0 & 1 & 0 \\
\hline 32618794 & Naqvi et al. [72] & June & USA & 1 & 35 & 22 & 28 & 1 & 1 & 1 & 0 & 1 & 9 & 0 \\
\hline 32702930 & Zheng et al. [73] & June & China & 2 & 31 & 37.5 & NA & 0 & 0 & 0 & 0 & 2 & 28.5 & 1 \\
\hline 32716009 & Douedi et al. [74] & June & USA & 3 & 23 & 29.5 & NA & NA & NA & NA & NA & 3 & 9 & 3 \\
\hline 32740456 & Marzollo et al. [75] & June & Italy & 1 & 29 & 38 & NA & 0 & 0 & 0 & 0 & 1 & 18 & 0 \\
\hline 32667391 & Reis et al. [76] & July & Brazil & 3 & 29.5 & 34 & NA & 0 & 0 & 0 & 0 & 3 & 18 & 2 \\
\hline 32675129 & Oliva et al. [77] & July & USA & 1 & 35 & 29 & NA & 1 & 0 & 0 & NA & 1 & 15 & 1 \\
\hline 32704477 & Richtmann et al. [78] & July & Brazil & 5 & 32 & 25.5 & 27.7 & 0 & 0 & 0 & 2 & 0 & NA & 0 \\
\hline 32714844 & Kolkova et al. [79] & July & Sweden & 1 & 27 & 32 & 57 & 1 & 0 & 0 & 1 & 1 & 31 & 1 \\
\hline 32715804 & Soleimani et al. [80] & July & Iran & 1 & 30 & 21 & 36 & 0 & 0 & 0 & 1 & 1 & 31 & 0 \\
\hline 32723092 & Easterlin et al. [81] & July & USA & 1 & 22 & 23 & NA & NA & NA & NA & NA & 1 & 28 & 1 \\
\hline 32754425 & Chong et al. [82] & July & USA & 1 & 41 & 32 & 35.6 & 0 & 0 & 0 & 1 & 1 & 12 & 1 \\
\hline 32784239 & Ahmed et al. [83] & July & UK & 1 & 26 & 37 & 25 & 1 & 1 & 0 & 0 & 0 & 7 & 0 \\
\hline 32773854 & Chhabra et al. [84] & May & India & 1 & 28 & 38 & 31.5 & 1 & 0 & 0 & 0 & 0 & 22 & 0 \\
\hline 32784234 & Figueiredo et al. [85] & July & Portugal & 1 & 35 & 39 & NA & 0 & 0 & 0 & 0 & 0 & 15 & 0 \\
\hline 32788159 & Federici et al. [86] & August & France & 1 & 33 & 23.5 & NA & 0 & 1 & 0 & NA & 1 & 19 & 1 \\
\hline 32791731 & Peng et al. [87] & June & China & 1 & 33 & 38 & NA & NA & NA & NA & NA & 0 & 5 & 0 \\
\hline Summary & & & & 64 & 31.10 & 32.72 & 35.80 & $\begin{array}{l}10 \\
(23.3)\end{array}$ & $\begin{array}{l}4 \\
(9.3)\end{array}$ & $\begin{array}{l}4 \\
(9.3)\end{array}$ & $\begin{array}{l}12 \\
(29.3)\end{array}$ & $\begin{array}{l}27 \\
(43.5)\end{array}$ & $\begin{array}{l}14.6 \\
(9.0)\end{array}$ & $\begin{array}{l}32 \\
(50.8)\end{array}$ \\
\hline
\end{tabular}

PMID PubMed identifier, $N$ number of subjects, GW gestational age measured in weeks, $B M I$ body mass index, DM diabetes mellitus, $H T$ hypertension, ICU intensive care unit, HLOS hospital length of stay, NA not available, UK United Kingdom, USA United States of America

difference in ICU admission and mechanical ventilation support.

\section{Subgroup analysis}

The ICU admission rate, average HLOS, and preterm birth rate were found to be consistently increased in studies utilizing high proportions of immunosuppressants and antibiotics. Studies with high ICU admission and preterm birth rates were more likely to observe with high proportions of oxygen support and symptomatic patients. Studies with more ICU admitted subjects had also reported more usage of HCQ and anticoagulants. Increased ICU admission was also observed in studies with more obese patients. The preterm birth rate was higher in studies with more cesarean sections, more use of zinc/magnesium, and DM and HTN subjects while the preterm birth rate was less likely to observe in studies with low use of HCQ and no use of plasma therapy.
The longest HLOS was noticed in studies using plasma therapy. Studies with fewer HTN patients had also reported decreased HLOS (Table 4).

\section{Systematic review of case reports}

The average age of patients was found to be similar among geographic locations while other maternal characteristics were found to be different across geographic locations (Table 5). The most common treatments in Asian studies were antibiotics (80\%), antivirals (60\%), oxygen support (40\%), and steroids (40\%) with the least usage of immunosuppressants (4\%). In the US studies, the most frequent therapeutics were oxygen support (73\%), steroids (71\%), antibiotics (53\%), antivirals (47\%), and HCQ (40\%). In European studies, oxygen support (69\%), antibiotics (62\%), steroids (46\%), and zinc/magnesium (38\%) were commonly used. HCQ (44\%) and anticoagulants (22\%) were frequently used in Latin 
Table 2 Overall estimates of maternal characteristics, treatment profile, and outcomes

\begin{tabular}{|c|c|c|c|}
\hline & Case series & Case reports & $P$-value \\
\hline & Proportion $(95 \% \mathrm{Cl})$ & Proportion $(95 \% \mathrm{Cl})$ & \\
\hline Maternal Characteristics & & & \\
\hline Age (years) mean (95\% Cl) & $30.60(28.90,32.40)^{a}$ & $31.10(29.97,32.24)$ & 0.359 \\
\hline BMI $\left(\mathrm{Kg} / \mathrm{m}^{2}\right)$ mean $(95 \% \mathrm{Cl})$ & $29.40(27.30,31.70)^{a}$ & $35.81(32.04,39.57)$ & $<0.001$ \\
\hline Gestational age (weeks) mean (95\% Cl) & $31.30(28.80,33.80)^{a}$ & $32.70(31.31,34.24)$ & 0.050 \\
\hline Symptomatic presentation & $0.89(0.75,0.98)$ & $0.89(0.78,0.95)$ & 0.006 \\
\hline Comorbidities & & & \\
\hline Diabetes mellitus & $0.04(0.03,0.07)$ & $0.23(0.12,0.39)$ & $<0.001$ \\
\hline Hypertension & $0.04(0.02,0.07)$ & $0.09(0.03,0.22)$ & 0.283 \\
\hline Asthma & $0.04(0.02,0.06)$ & $0.09(0.03,0.22)$ & 0.131 \\
\hline Obesity & $0.09(0.04,0.15)$ & $0.29(0.16,0.46)$ & $<0.001$ \\
\hline Treatment & & & \\
\hline Oxygen support & $0.33(0.20,0.47)$ & $0.53(0.40,0.66)$ & $<0.001$ \\
\hline Steroids & $0.06(0.00,0.19)$ & $0.44(0.31,0.57)$ & $<0.001$ \\
\hline Immunosuppressants & $0.01(0.00,0.03)$ & $0.10(0.04,0.20)$ & 0.004 \\
\hline Hydroxychloroquine & $0.10(0.03,0.19)$ & $0.26(0.16,0.38)$ & 0.082 \\
\hline Antivirals & $0.33(0.18,0.49)$ & $0.45(0.32,0.58)$ & $<0.001$ \\
\hline Zinc/Magnesium & $0.02(0.00,0.14)$ & $0.21(0.12,0.34)$ & 0.007 \\
\hline Anticoagulants & $0.03(0.00,0.07)$ & $0.12(0.05,0.23)$ & 0.031 \\
\hline Antibiotics & $0.36(0.21,0.52)$ & $0.64(0.51,0.76)$ & $<0.001$ \\
\hline Plasma therapy/Anti-liver damage & $0.02(0.00,0.06)$ & $0.14(0.07,0.26)$ & $<0.001$ \\
\hline Mechanical ventilation & $0.03(0.01,0.07)$ & $0.35(0.23,0.49)$ & $<0.001$ \\
\hline Maternal \& Pregnancy Outcomes & & & \\
\hline ICU admission & $0.06(0.02,0.10)$ & $0.43(0.31,0.57)$ & $<0.001$ \\
\hline Hospital length of stay (days) mean ( $95 \%$ Cl) & $8.47(5.96,10.97)^{a}$ & $14.6(12.0,17.2)$ & $<0.001$ \\
\hline Maternal death & $0.003(0.00,0.02)$ & $0.05(0.01,0.13)$ & 0.108 \\
\hline Cesarean section & $0.62(0.54,0.71)$ & $0.69(0.56,0.80)$ & 0.085 \\
\hline Fetal demise & $<1 \%$ & $0.08(0.03,0.19)$ & 0.003 \\
\hline Neonatal death & $<1 \%$ & $0.02(0.00,0.08)$ & 0.143 \\
\hline Premature birth & $0.26(0.19,0.34)$ & $0.50(0.37,0.63)$ & $<0.001$ \\
\hline
\end{tabular}

${ }^{a}$ Weighted mean reported; $l^{2} \%$ measure of heterogeneity, $C l$ confidence interval, $I C U$ intensive care unit, $N A$ not applicable, $B M I$ body mass index

American studies than in rest countries. The mechanical ventilation and ICU admission were most commonly observed in the US (67 and 80\%) than European (38 and $38 \%$ ), Latin American (33 and 33\%), and Asian (16 and 28\%) studies, respectively. However, the average HLOS was highest in Asian (19.1 days) and Latin American studies (14.2 days) while similar in the US and European studies (12.6 and 11 days respectively). The preterm birth rate was highest in the US (69\%) followed by Europe (54\%), Asia (44\%) and Latin America (33\%). The cesarean section rate was highest in Latin American studies $(78 \%)$ but similar in other countries (68-69\%). The other maternal outcomes were minimally observed in any country except the higher maternal death (33\%) and fetal demise (56\%) reported in Latin American studies. In association analysis (Supplementary Table 3), the use of antivirals and oxygen support was consistently associated with all adverse outcomes. In addition, HCQ and immunosuppressant treatments were associated with an increased proportion of ICU admission while the use of zinc/magnesium and steroids was associated with the increased preterm birth rate.

\section{Discussion}

In the meta-analysis of average-risk patients, the US studies revealed better maternal outcomes with more asymptomatic patients, more comorbidities, and less use of overall treatment interventions compared to the Asian and European countries. Furthermore, the average HLOS was also shorter in the US and European studies than in Asian 
Table 3 Meta-analysis of maternal characteristics, therapeutics, and outcomes by geographic location

\begin{tabular}{|c|c|c|c|c|c|c|c|c|c|}
\hline & \multicolumn{3}{|c|}{ Asia } & \multicolumn{3}{|c|}{ USA } & \multicolumn{3}{|c|}{ Europe } \\
\hline & $\bar{N}$ & $1^{2}$ & $\begin{array}{l}\text { Proportion } \\
(95 \% \mathrm{Cl})\end{array}$ & $\bar{N}$ & $1^{2}$ & $\begin{array}{l}\text { Proportion } \\
(95 \% \mathrm{Cl})\end{array}$ & $\bar{N}$ & $1^{2}$ & $\begin{array}{l}\text { Proportion } \\
(95 \% \mathrm{Cl})\end{array}$ \\
\hline \multicolumn{10}{|l|}{ Maternal Characteristics } \\
\hline Age (years); mean $(95 \% \mathrm{Cl})^{\mathrm{a}}$ & 10 & NA & $28.40(24.80,32.10)$ & 5 & NA & $30.80(29.60,31.90)$ & 7 & NA & $30.60(28.90,32.40)$ \\
\hline BMI $\left(\mathrm{kg} / \mathrm{m}^{2}\right)$; mean $(95 \% \mathrm{Cl})^{\mathrm{a}}$ & 1 & NA & 27.00 (single study) & 4 & NA & $30.60(30.40,31.00)$ & 5 & NA & $29.40(27.30,31.70)$ \\
\hline Gestational age (weeks); mean $(95 \% \mathrm{Cl})^{\mathrm{a}}$ & 9 & NA & $34.80(32.40,37.20)$ & 4 & NA & $31.90(26.20,37.70)$ & 8 & NA & $31.30(28.80,33.80)$ \\
\hline Symptomatic Presentation & 6 & 0.0 & $1.00(0.96,1.00)$ & 4 & 97.1 & $0.54(0.21,0.85)$ & 8 & 93.4 & $0.92(0.80,0.99)$ \\
\hline \multicolumn{10}{|l|}{ Comorbidities } \\
\hline Diabetes mellitus & 6 & 46.1 & $0.07(0.02,0.14)$ & 5 & 26.0 & $0.06(0.03,0.09)$ & 6 & 45.2 & $0.03(0.01,0.06)$ \\
\hline Hypertension & 6 & 16.5 & $0.03(0.00,0.07)$ & 5 & 82.8 & $0.07(0.01,0.15)$ & 6 & 21.8 & $0.03(0.01,0.06)$ \\
\hline Asthma & 6 & 0.0 & $0.00(0.00,0.01)$ & 5 & 24.2 & $0.07(0.04,0.11)$ & 6 & 79.6 & $0.05(0.01,0.11)$ \\
\hline Obesity & 5 & 0.0 & $0.00(0.00,0.03)$ & 3 & ID & $0.20(0.10,0.32)$ & 5 & 64.8 & $0.11(0.06,0.19)$ \\
\hline \multicolumn{10}{|l|}{ Treatment } \\
\hline Oxygen support & 9 & 94.9 & $0.55(0.20,0.88)$ & 5 & 59.0 & $0.07(0.03,0.12)$ & 6 & 84.2 & $0.33(0.21,0.46)$ \\
\hline Steroids & 9 & 73.5 & $0.05(0.00,0.16)$ & 4 & 83.8 & $0.01(0.00,0.09)$ & 8 & 98.8 & $0.10(0.00,0.44)$ \\
\hline Immunosuppressants & 9 & 0.0 & $0.00(0.00,0.02)$ & 4 & 87.9 & $0.02(0.00,0.11)$ & 7 & 83.3 & $0.02(0.00,0.07)$ \\
\hline Hydroxychloroquine & 9 & 43.2 & $0.00(0.00,0.04)$ & 5 & 84.0 & $0.07(0.02,0.17)$ & 8 & 96.6 & $0.26(0.09,0.49)$ \\
\hline Antivirals & 9 & 89.8 & $0.80(0.56,0.97)$ & 4 & 59.1 & $0.01(0.00,0.05)$ & 8 & 89.8 & $0.12(0.04,0.22)$ \\
\hline Zinc/Magnesium & 9 & 78.5 & $0.02(0.00,0.11)$ & 4 & 0.0 & $0.00(0.00,0.01)$ & 8 & 99.0 & $0.05(0.00,0.39)$ \\
\hline Anticoagulant & 9 & 67.9 & $0.02(0.00,0.09)$ & 4 & 92.2 & $0.03(0.00,0.17)$ & 8 & 91.8 & $0.04(0.00,0.12)$ \\
\hline Antibiotics & 9 & 95.4 & $0.78(0.41,1.00)$ & 5 & 84.3 & $0.12(0.04,0.22)$ & 8 & 94.1 & $0.14(0.04,0.29)$ \\
\hline Plasma therapy/Anti-liver damage & 9 & 93.1 & $0.06(0.00,0.3)$ & 5 & 20.3 & $0.00(0.00,0.01)$ & 8 & 81.3 & $0.01(0.00,0.04)$ \\
\hline Mechanical ventilation & 9 & 86.8 & $0.04(0.00,0.18)$ & 4 & 84.6 & $0.02(0.00,0.11)$ & 8 & 0.0 & $0.06(0.04,0.08)$ \\
\hline \multicolumn{10}{|l|}{ Maternal \& Pregnancy Outcomes } \\
\hline ICU admission & 8 & 87.9 & $0.05(0.00,0.23)$ & 4 & 88.8 & $0.06(0.00,0.19)$ & 8 & 52.7 & $0.07(0.04,0.11)$ \\
\hline HLOS (days) mean $(95 \% \mathrm{Cl})^{\mathrm{a}}$ & 2 & NA & $11.86(8.55,15.17)$ & 4 & NA & $9.40(2.96,15.83)$ & 5 & NA & $7.34(5.46,9.23)$ \\
\hline Maternal death & 10 & 71.5 & $0.01(0.00,0.07)$ & 5 & 20.3 & $0.00(0.00,0.01)$ & 8 & 32.9 & $0.01(0.00,0.02)$ \\
\hline Cesarean section & 10 & 85.1 & $0.80(0.61,0.94)$ & 5 & 0.0 & $0.46(0.39,0.53)$ & 8 & 72.3 & $0.53(0.41,0.66)$ \\
\hline Fetal demise & 2 & ID & $0.02(0.00,0.05)$ & 2 & ID & $0.01(0,0.04)$ & 1 & ID & $0.02(0.00,0.10)$ \\
\hline Premature birth & 8 & 77.8 & $0.35(0.17,0.55)$ & 5 & 0.0 & $0.13(0.09,0.18)$ & 6 & 65.1 & $0.29(0.20,0.40)$ \\
\hline
\end{tabular}

Note: ${ }^{2}$ Weighted mean reported; Asia includes China, India and Iran; Europe includes France, Italy, Spain and Turkey

$I^{2} \%$ measure of heterogeneity, $C I$ confidence interval, ICU intensive care unit, HLOS hospital length of stay, ID insufficient data, NA not applicable, BMI body mass index, USA United States of America

studies without much increase in ICU admission or mechanical ventilation use. Over the course of the pandemic time-lapse, most therapeutic use for COVID-19 showed a declining pattern. The subgroup analysis showed that antibiotics and immunosuppressants were consistently associated with adverse outcomes in average-risk patients, while antivirals and oxygen support were associated with all adverse outcomes in severe cases. In addition, HCQ was also associated with more ICU admission in both average-risk and severe cases.

Our analysis of case reports and case series studies showed marked differences in clinical presentation and therapeutics. As case studies often report complex cases requiring critical management [19], our analysis of case reports also present therapeutics and outcomes of severe cases compared to case series analysis. Furthermore, the US and European studies presented more critically ill patients in their case reports compared to Asian and Latin American studies. The average age of pregnant patients was the early $30 \mathrm{~s}$ in both case reports and case series analyses. Some recent reports identified multiple reasons for more COVID-19 detection in younger persons than older adults [88]. Recently a study showed that pregnant women with COVID-19 were more likely to be in the age of 25-34 years compared to non-pregnant women with COVID-19 [6]. As observed in our previous study [19], the US patients were more likely to be asymptomatic compared to the Asian and European patients. This 


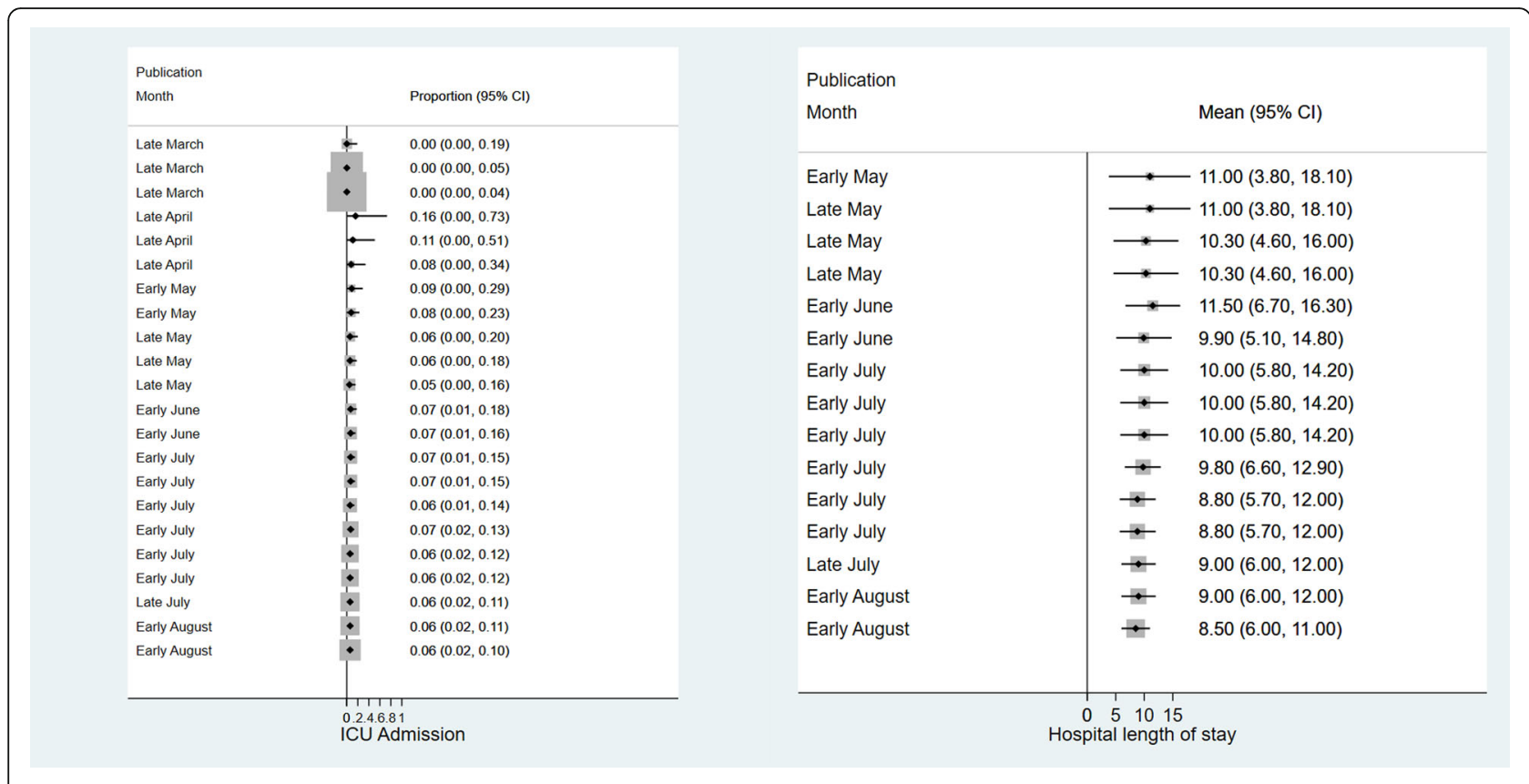

Fig. 2 Pattern of intensive care unit admission and hospital length of stay of pregnant women infected with SARS-CoV-2 in case series studies

could be due to more availability of testing of COVID19 in pregnant women coming in for a routine follow up in the US. Breslin et al. [89] presented the importance of early screening for pregnant patients and their positive outcomes using a retrospective chart review. The ICU admission and preterm birth rates in our study were comparable to other studies [19, 90]. However, multiple studies $[6,91]$ have shown that pregnant women with COVID-19 were more likely to have hospitalization, ICU admission, mechanical ventilation use compared to nonpregnant women with COVID-19. Moreover, we also previously showed high rates of adverse pregnancy

\begin{tabular}{|c|c|c|c|c|}
\hline \multicolumn{2}{|l|}{ Publication } & \multicolumn{3}{|l|}{ Publication } \\
\hline Month & Proportion (95\% Cl) & \multicolumn{2}{|l|}{ Month } & \multirow{2}{*}{$\begin{array}{l}\text { Proportion }(95 \% \mathrm{Cl}) \\
0.27(0.10,0.57)\end{array}$} \\
\hline Early March & $\longrightarrow 0.91(0.62,0.98)$ & Early March & $\rightarrow-$ & \\
\hline Late March & $\rightarrow 0.89(0.73,0.99)$ & Late March & $\rightarrow$ & $0.58(0.38,0.76)$ \\
\hline Late March & $\leftarrow 0.92(0.81,0.99)$ & Late March & $\longrightarrow$ & $0.37(0.03,0.80)$ \\
\hline Late March & $\rightarrow 0.94(0.85,0.99)$ & Late March & $\longrightarrow$ & $0.38(0.09,0.72)$ \\
\hline Late April & $\rightarrow 0.93(0.84,0.99)$ & Late April & $\longrightarrow$ & $0.47(0.17,0.78)$ \\
\hline Late April & $\longrightarrow 0.83(0.56,0.99)$ & Late April & $\longrightarrow$ & $0.47(0.17,0.78)$ \\
\hline Late April & $\longrightarrow-0.77(0.50,0.96)$ & Late April & $\rightarrow$ & $0.40(0.16,0.67)$ \\
\hline Early May & $\longrightarrow \quad 0.72(0.47,0.92)$ & Early May & $\rightarrow$ & $0.36(0.17,0.57)$ \\
\hline Early May & $\rightarrow \quad 0.69(0.46,0.88)$ & Early May & $\rightarrow$ & $0.34(0.17,0.53)$ \\
\hline Late May & $\rightarrow \quad 0.69(0.49,0.87)$ & Late May & $\rightarrow$ & $0.32(0.17,0.48)$ \\
\hline Late May & $\rightarrow \quad 0.72(0.52,0.89)$ & Late May & $\rightarrow$ & $0.32(0.17,0.48)$ \\
\hline Late May & $\rightarrow \quad 0.75(0.55,0.90)$ & Late May & $\rightarrow$ & $0.30(0.16,0.45)$ \\
\hline Early June & $\rightarrow \quad 0.72(0.54,0.87)$ & Early June & 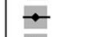 & $0.28(0.16,0.42)$ \\
\hline Early June & $\rightarrow \quad 0.70(0.53,0.85)$ & Early June & $\rightarrow$ & $0.28(0.16,0.42)$ \\
\hline Early July & $\mp \quad 0.70(0.55,0.84)$ & Early July & $\rightarrow$ & $0.28(0.16,0.40)$ \\
\hline Early July & $\rightarrow \quad 0.68(0.54,0.81)$ & Early July & $\rightarrow$ & $0.26(0.16,0.37)$ \\
\hline Early July & $\rightarrow \quad 0.67(0.54,0.80)$ & Early July & $\rightarrow$ & $0.26(0.17,0.36)$ \\
\hline Early July & $0.66(0.54,0.78)$ & Early July & $\rightarrow$ & $0.29(0.18,0.40)$ \\
\hline Early July & $0.65(0.53,0.75)$ & Early July & $\rightarrow$ & $0.27(0.18,0.37)$ \\
\hline Early July & $0.63(0.53,0.74)$ & Early July & $\rightarrow$ & $0.27(0.18,0.36)$ \\
\hline Late July & $0.63(0.53,0.73)$ & Late July & $\leftarrow$ & $0.26(0.18,0.35)$ \\
\hline Early August & $0.62(0.53,0.71)$ & Early August & $\rightarrow$ & $0.26(0.18,0.35)$ \\
\hline Early August & $\leftarrow \quad 0.62(0.54,0.71)$ & Early August & $\leftarrow$ & $0.26(0.19,0.34)$ \\
\hline \multicolumn{2}{|c|}{ 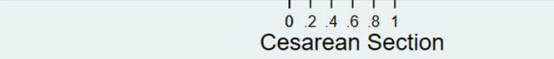 } & \multicolumn{3}{|c|}{$\begin{array}{cccccc}0 & 2 & 1 & 1 & 1 & 1 \\
0 & 2 & 4 & 6 & .8 & 1 \\
\text { Premature Birth }\end{array}$} \\
\hline
\end{tabular}




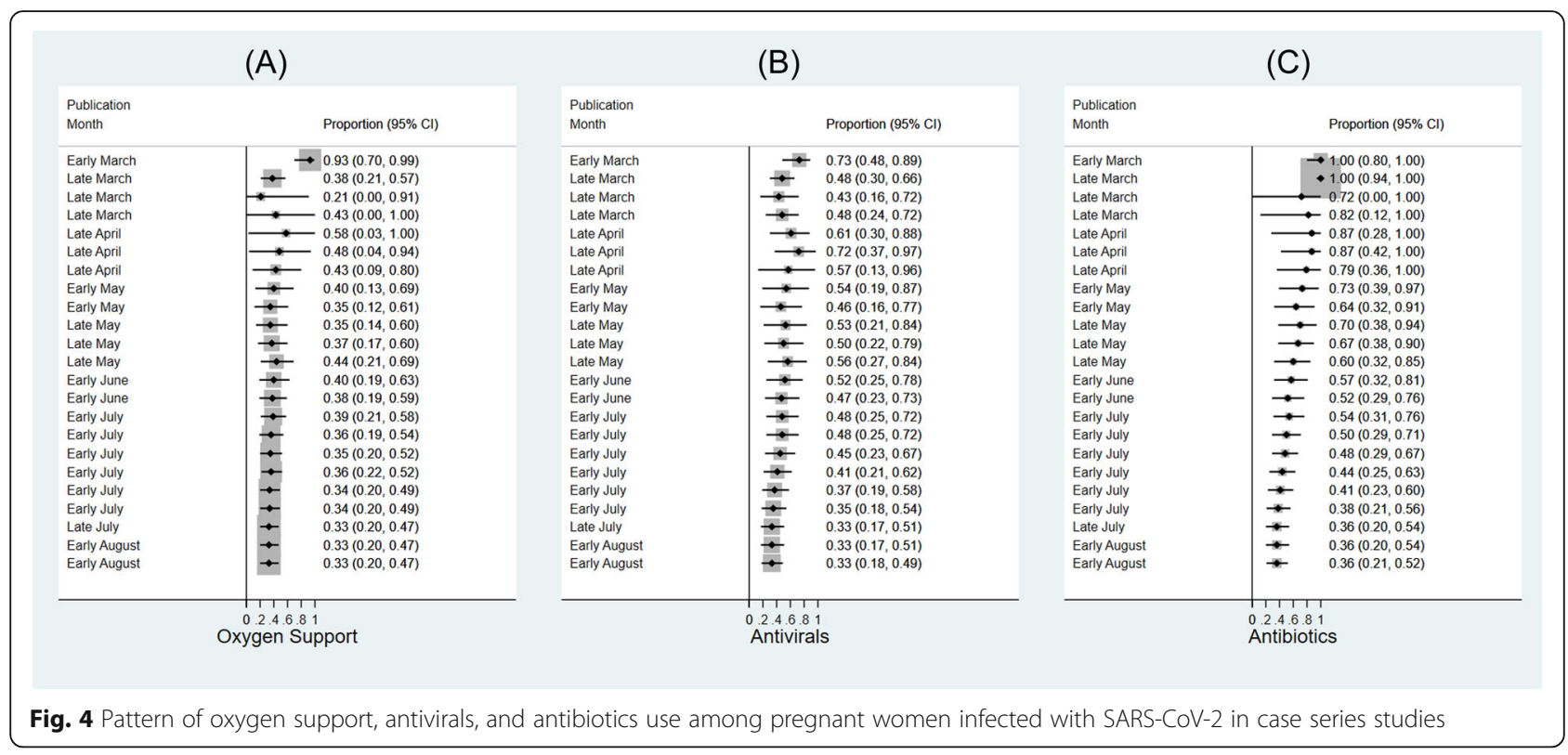

outcomes including preterm and cesarean section outcomes in pregnant women with COVID-19 [19] suggesting a scope of improvements in managing pregnant women.

We observed that antivirals and antibiotics were mostly used in Asian studies for managing all pregnant patients compared to other countries. Given the lack of efficacy data for most therapeutics of COVID-19 [92], the practice in the US and European countries might have been to minimally expose any therapeutics to average-risk pregnant patients. The highest use of antibiotics in Asian studies may be due to local guidelines for managing COVID-19 patients [93], suspicion of bacterial or fungal coinfections due to unavailability of rapid and affordable testing to differentiate viral and bacterial infections, and health-care-associated infections [94] due to prolonged hospitalization. In our analysis, a higher than anticipated use of antibiotics was also observed in the US and European studies. In a US study, more than half of the patients initially received antibiotics for the suspicion of bacterial infection, later in their stay, testing revealed that more than $96 \%$ of patients had the COVID-19 only and did not need antibiotics demonstrating their overuse [14]. Our study suggests that indiscriminate use of antibiotics needs to be minimized particularly in pregnant patients.

Our study showed a consistent pattern in the increased rates of ICU admission and preterm birth including longer HLOS associated with the increased use of antibiotics in average-risk patients. This observation could be due to health-care-associated infections requiring more antibiotics in ICU and hospitalized patients [94]. In addition, bacterial infections during pregnancy have been associated with an increased risk of preterm birth [95]. Although the association between the increased use of antibiotics in COVID-19 patients and antimicrobial resistance (AMR) is not clear [96], there may be likely to observe more AMR among COVID-19 patients. This may exacerbate the management of drugresistant patients who are at-risk for bacterial infections. Though immunosuppressant was minimally used in the US and European studies, it was found to be associated with adverse clinical and maternal outcomes in our analysis. This finding was further supported by the analysis of case reports of the US studies. The use of immunosuppressants may yield significant complications and requires a proper assessment prior to its use [97]. Immunosuppressant use may be avoided in pregnant women unless it is clinically indicated.

The use of antivirals was associated with increased adverse outcomes in the analysis of case reports. Relatively lower use of antivirals in the US and European studies may be one of the reasons for having shorter HLOS compared to Asian studies. There have been studies where mixed effects of antivirals have been observed with potentially no benefits $[98,99]$. As oxygen supplementation is required in the majority of critically ill patients [100], we also observed a high rate of ICU admission and preterm birth with a high proportion of oxygen support both in average-risk and severe cases of COVID-19. Although the reason for the potential association between oxygen therapy and preterm birth is unclear, the use of oxygen therapy was associated with an increased use of COVID-19 specific medications and inpatient mortality [101]. Given no evidence of optimal strategy for oxygen therapy in COVID-19 patients [99], a careful consideration is needed for oxygen therapy in 
Table 4 Meta-analysis of outcomes according to maternal and treatments characteristics

\begin{tabular}{|c|c|c|c|c|}
\hline \multirow{3}{*}{ Treatment } & \multirow[t]{2}{*}{ Groups } & \multirow{2}{*}{$\begin{array}{l}\text { ICU Admission } \\
\text { Proportion } \\
(95 \% \mathrm{Cl})\end{array}$} & \multirow{2}{*}{$\begin{array}{l}\text { Hospital Length of Stay } \\
\text { Mean }(95 \% \mathrm{Cl})^{\mathrm{a}}\end{array}$} & \multirow{2}{*}{$\begin{array}{l}\text { Preterm Birth } \\
\text { Proportion } \\
(95 \% \mathrm{Cl})\end{array}$} \\
\hline & & & & \\
\hline & & & & \\
\hline \multirow[t]{2}{*}{ Oxygen support } & $\leq 0.25$ (low) & $0.03(0.00,0.08)$ & $8.89(4.31,13.48)$ & $0.20(0.10,0.33)$ \\
\hline & $>0.25$ (high) & $0.10(0.02,0.21)$ & $9.10(8.05,10.15)$ & $0.33(0.21,0.46)$ \\
\hline \multirow[t]{2}{*}{ Steroids } & Not given & $0.06(0.01,0.15)$ & $7.45(2.22,12.69)$ & $0.29(0.17,0.42)$ \\
\hline & Given & $0.06(0.02,0.11)$ & $9.18(6.49,11.86)$ & $0.27(0.17,0.37)$ \\
\hline \multirow[t]{2}{*}{ Immunosuppressants } & Not given & $0.03(0.01,0.06)$ & $7.75(5.10,10.41)$ & $0.26(0.18,0.36)$ \\
\hline & Given & $0.24(0.05,0.51)$ & $12.37(4.22,20.52)$ & $0.35(0.13,0.60)$ \\
\hline \multirow[t]{2}{*}{ Hydroxychloroquine } & $\leq 0.043$ (low) & $0.02(0.00,0.05)$ & $9.95(6.24,13.65)$ & $0.30(0.16,0.46)$ \\
\hline & > 0.043 (high) & $0.11(0.04,0.21)$ & $7.30(4.22,10.38)$ & $0.22(0.15,0.30)$ \\
\hline \multirow[t]{2}{*}{ Antivirals } & $\leq 0.25$ (low) & $0.05(0.02,0.09)$ & $8.84(5.63,12.05)$ & $0.29(0.16,0.44)$ \\
\hline & $>0.25$ (high) & $0.07(0.00,0.20)$ & $7.38(4.67,10.10)$ & $0.25(0.16,0.34)$ \\
\hline \multirow[t]{2}{*}{ Zinc/Magnesium } & Not given & $0.06(0.02,0.11)$ & $8.34(5.36,11.32)$ & $0.26(0.18,0.34)$ \\
\hline & Given & $0.07(0.03,0.13)$ & NA & $0.44(0.30,0.58)$ \\
\hline \multirow[t]{2}{*}{ Anticoagulants } & Not given & $0.03(0.02,0.05)$ & $8.10(4.98,11.23)$ & $0.27(0.18,0.38)$ \\
\hline & Given & $0.18(0.03,0.40)$ & $9.24(3.73,14.74)$ & $0.28(0.14,0.44)$ \\
\hline \multirow[t]{2}{*}{ Antibiotics } & $\leq 0.23$ (low) & $0.04(0.01,0.06)$ & $6.37(3.49,9.24)$ & $0.22(0.13,0.32)$ \\
\hline & $>0.23$ (high) & $0.09(0.02,0.20)$ & $10.44(6.23,14.65)$ & $0.31(0.20,0.44)$ \\
\hline \multirow[t]{2}{*}{ Plasma/Anti-Liver damage } & Not given & $0.06(0.02,0.11)$ & $7.74(5.26,10.22)$ & $0.28(0.20,0.36)$ \\
\hline & Given & $0.04(0.00,0.22)$ & $13.99(9.27,18.70)$ & $0.17(0.05,0.32)$ \\
\hline \multirow[t]{2}{*}{ Cesarean section } & $\leq 0.51$ (low) & $0.06(0.02,0.12)$ & $8.73(5.11,12.34)$ & $0.21(0.13,0.30)$ \\
\hline & $>0.51$ (high) & $0.06(0.00,0.15)$ & $7.95(5.45,10.45)$ & $0.33(0.20,0.46)$ \\
\hline \multirow[t]{2}{*}{ Symptomatic } & $\leq 0.94$ (low) & $0.04(0.01,0.08)$ & $7.62(3.80,11.45)$ & $0.18(0.15,0.22)$ \\
\hline & > 0.94 (high) & $0.10(0.02,0.22)$ & $7.85(5.17,10.53)$ & $0.38(0.23,0.54)$ \\
\hline \multicolumn{5}{|l|}{ Comorbidities } \\
\hline \multirow[t]{2}{*}{ Diabetes mellitus } & $\leq 0.06$ (low) & $0.07(0.02,0.13)$ & $8.32(5.08,11.55)$ & $0.21(0.14,0.29)$ \\
\hline & $>0.06$ (high) & $0.08(0.00,0.22)$ & $8.62(4.42,12.82)$ & $0.39(0.19,0.61)$ \\
\hline \multirow[t]{2}{*}{ Hypertension } & $\leq 0.03$ (low) & $0.11(0.01,0.27)$ & $10.81(6.60,15.01)$ & $0.27(0.12,0.44)$ \\
\hline & $>0.03$ (high) & $0.04(0.02,0.07)$ & $6.76(4.54,8.98)$ & $0.32(0.18,0.47)$ \\
\hline \multirow[t]{2}{*}{ Asthma } & $\leq 0.034$ (low) & $0.07(0.00,0.22)$ & $9.97(5.63,14.30)$ & $0.35(0.19,0.53)$ \\
\hline & > 0.034 (high) & $0.07(0.03,0.13)$ & $7.28(3.95,10.62)$ & $0.24(0.11,0.40)$ \\
\hline \multirow[t]{2}{*}{ Obesity } & $\leq 0.10$ (low) & $0.04(0.01,0.08)$ & $7.71(3.58,11.85)$ & $0.42(0.10,0.78)$ \\
\hline & > 0.10 (high) & $0.13(0.03,0.29)$ & $8.25(4.37,12.13)$ & $0.33(0.15,0.53)$ \\
\hline
\end{tabular}

${ }^{a}$ Weighted mean reported; ICU intensive care unit, $\mathrm{Cl}$ confidence interval, NA not available; bold values indicate a significant presence (at least $5 \%$ ) of the condition relative to their average value

pregnant patients. Similar to our study findings, HCQ has been associated with an increased risk of ICU admission [102] and the use of HCQ may be avoided in pregnant women from western countries. Consistent with our study, findings from multiple clinical trials indicated benefits from steroid use in COVID-19 cases and steroids have been recommended for treating severe and critical COVID-19 cases [11]. The anticoagulants were given rarely but mostly to ICU patients in both case series as well as case reports studies as it has been recommended for high-risk individuals only [15]. In our study, plasma therapy was found to be associated with longer HLOS which could be due to its emergency use authorization for hospitalized patients only. Patients who received zinc/magnesium had shorter HLOS in case reports but associated with increased preterm birth in all patients. The evidence is emerging for the potential benefits of zinc/magnesium in COVID-19 and it seems promising in cases who are at low risk of preterm birth [103, 104]. 
Table 5 Maternal characteristics, treatments, and outcomes among the 64 pregnant women by geographic locations

\begin{tabular}{|c|c|c|c|c|}
\hline & Asia & USA & Europe & Latin America \\
\hline & $\begin{array}{l}\text { Proportion } \\
(95 \% \mathrm{Cl})\end{array}$ & $\begin{array}{l}\text { Proportion } \\
(95 \% \mathrm{Cl})\end{array}$ & $\begin{array}{l}\text { Proportion } \\
(95 \% \mathrm{Cl})\end{array}$ & $\begin{array}{l}\text { Proportion } \\
(95 \% \mathrm{Cl})\end{array}$ \\
\hline \multicolumn{5}{|l|}{ Maternal Characteristics } \\
\hline Age (years); mean (95\% Cl) & $30.00(28.70,31.20)$ & $30.90(27.30,34.50)$ & $32.8(30.10,35.40)$ & $32.2(29.50,34.90)$ \\
\hline BMI $\left(\mathrm{kg} / \mathrm{m}^{2}\right)$; mean $(95 \% \mathrm{Cl})$ & $33.70(5.20,62.30)$ & $37.0(30.20,43.90)$ & $41.4(32.70,50.10)$ & $28.9(25.80,32.00)$ \\
\hline Gestational age (weeks); mean (95\% Cl) & $36.20(34.60,37.80)$ & $28.40(25.50,31.30)$ & $33.4(30.50,36.30)$ & $29.2(25.80,32.50)$ \\
\hline Symptomatic Presentation & $0.80(0.59,0.93)$ & $1.00(0.77,1.00)$ & $1.00(0.75,1.00)$ & $0.78(0.40,0.97)$ \\
\hline \multicolumn{5}{|l|}{ Comorbidities } \\
\hline Diabetes mellitus & $0.09(0.00,0.41)$ & $0.40(0.12,0.74)$ & $0.38(0.14,0.68)$ & $0.00(0.00,0.34)$ \\
\hline Hypertension & $0.00(0.00,0.28)$ & $0.10(0.00,0.45)$ & $0.23(0.05,0.54)$ & $0.00(0.00,0.34)$ \\
\hline Asthma & $0.00(0.00,0.28)$ & $0.40(0.12,0.74)$ & $0.00(0.00,0.25)$ & $0.00(0.00,0.34)$ \\
\hline Obesity & $0.09(0.00,0.41)$ & $0.56(0.21,0.86)$ & $0.25(0.05,0.57)$ & NA \\
\hline \multicolumn{5}{|l|}{ Treatment Profiles } \\
\hline Oxygen support & $0.40(0.21,0.61)$ & $0.73(0.45,0.92)$ & $0.69(0.39,0.91)$ & $0.33(0.07,0.70)$ \\
\hline Steroids & $0.40(0.21,0.61)$ & $0.71(0.42,0.92)$ & $0.46(0.19,0.75)$ & $0.11(0.00,0.48)$ \\
\hline Immunosuppressants & $0.04(0.00,0.21)$ & $0.33(0.12,0.62)$ & $0.00(0.00,0.25)$ & $0.00(0.00,0.34)$ \\
\hline Hydroxychloroquine & $0.13(0.03,0.32)$ & $0.40(0.16,0.68)$ & $0.23(0.05,0.54)$ & $0.44(0.14,0.79)$ \\
\hline Antivirals & $0.60(0.39,0.79)$ & $0.47(0.21,0.73)$ & $0.23(0.05,0.54)$ & $0.33(0.07,0.70)$ \\
\hline Zinc/Magnesium & $0.12(0.03,0.31)$ & $0.36(0.13,0.65)$ & $0.38(0.14,0.68)$ & $0.00(0.00,0.34)$ \\
\hline Anticoagulants & $0.08(0.01,0.26)$ & $0.08(0.00,0.38)$ & $0.15(0.02,0.45)$ & $0.22(0.03,0.60)$ \\
\hline Antibiotics & $0.80(0.59,0.93)$ & $0.53(0.27,0.79)$ & $0.62(0.32,0.86)$ & $0.44(0.14,0.79)$ \\
\hline Plasma therapy/Anti-liver damage & $0.20(0.07,0.41)$ & $0.20(0.04,0.48)$ & $0.08(0.00,0.36)$ & $0.00(0.00,0.34)$ \\
\hline Mechanical ventilation & $0.16(0.05,0.36)$ & $0.67(0.38,0.88)$ & $0.38(0.14,0.68)$ & $0.33(0.07,0.70)$ \\
\hline \multicolumn{5}{|l|}{ Maternal \& Pregnancy Outcomes } \\
\hline ICU admission & $0.28(0.12,0.49)$ & $0.80(0.52,0.96)$ & $0.38(0.14,0.68)$ & $0.33(0.07,0.70)$ \\
\hline HLOS (days) mean (95\% Cl) & $19.10(13.50,24.80)$ & $12.60(9.50,15.70)$ & $11.00(5.00,17.00)$ & $14.20(2.30,26.20)$ \\
\hline Cesarean section & $0.68(0.46,0.85)$ & $0.67(0.38,0.88)$ & $0.69(0.39,0.91)$ & $0.78(0.40,0.97)$ \\
\hline Maternal death & $0.00(0.00,0.14)$ & $0.00(0.00,0.22)$ & $0.00(0.00,0.25)$ & $0.33(0.07,0.70)$ \\
\hline Fetal demise & $0.00(0.00,0.14)$ & $0.00(0.00,0.25)$ & $0.00(0.00,0.25)$ & $0.56(0.21,0.86)$ \\
\hline Premature birth & $0.44(0.24,0.65)$ & $0.69(0.41,0.89)$ & $0.54(0.25,0.81)$ & $0.33(0.07,0.70)$ \\
\hline Neonatal death & $0.04(0.00,0.20)$ & $0.00(0.00,0.21)$ & $0.00(0.00,0.23)$ & $0.00(0.00,0.34)$ \\
\hline
\end{tabular}

Note: Asia includes China, India, Iran, Jordon, and Korea; Europe includes France, Italy, Netherlands, Portugal, Spain, Sweden, Turkey, and United Kingdom; South America includes Brazil and Peru

Cl confidence interval, ICU intensive care unit, HLOS hospital length of stay, BMI body mass index, NA not available, USA United States of America

Like another study demonstrating a significant drop in most COVID-19 medications [101], our cumulative meta-analysis demonstrated considerable drop in most therapeutics and associated outcomes for COVID-19 in pregnant women. As health care professionals learned through their own experiences and evidence accumulated from clinical trials, the decline in the most common therapeutics associated with no clear evidence of benefits was observed. However, there is a scope for reduction in therapeutics for optimizing outcomes by risk stratification in pregnant patients. Our findings suggest that optimal outcomes may be achieved by avoiding unnecessary medications, minimizing therapeutics associated with adverse outcomes, and adopting other preventive measures.

\section{Strengths and limitations}

Our systematic review and meta-analysis have some limitations. The main limitation is primarily the lack of high-quality data in the included studies with smaller sample sizes. However, our analysis included the largest series of studies in pregnant women with COVID-19. The estimates obtained from case reports studies may be biased despite having a fair number 
of case reports. Although we have used random effects models for estimating any characteristics and results reported according to geographic location, a substantial presence of heterogeneity in the estimates might introduce bias in the estimates. In the absence of non-pregnant women in included studies, our estimates for the periodical changes in treatment apply to pregnant patients only. Although the associations are mostly correlative not causative in this study, the observational evidence obtained from this study is useful for improving health care for pregnant patients. However, the results from subgroup and association analyses should be interpretated with cautions due to small sample sizes, complex interactions among treatments, and unadjusted analyses. Despite these limitations, our study is the first comprehensive study that provides the current therapeutic profile, their geographical distribution and chronological evaluation among pregnant women with COVID-19. We also associated the maternal and clinical outcomes with the current therapeutic use along with other maternal characteristics. Our study for the first time provides the geographical differences in maternal characteristics with therapeutics and pregnancy and clinical outcomes separately for average and severe cases of COVID-19.

\section{Conclusion}

In summary, a considerable decline in preterm birth rate and average HLOS was observed over the pandemic period. The rates of ICU admission, preterm birth, and average HLOS were estimated to be relatively higher in pregnant women with COVID-19 worldwide and varied by geographic locations. Although a considerable decline in the use of antibiotics, antivirals, oxygen support, and immunosuppressants was noticed, minimizing the use of these therapeutics by risk stratification and careful consideration may further improve maternal and clinical outcomes. More evidence is required for the use of steroids, zinc/magnesium, and plasma therapy in pregnant women with COVID-19. Geographical differences in therapeutics with differential rates of maternal and clinical outcomes in both average-risk and severe COVID-19 cases were observed. Overall avoiding unnecessary treatments and early screening of asymptomatic pregnant women particularly in their 30s may minimize adverse consequences of COVID-19.

\section{Abbreviations}

HCQ: Hydroxychloroquine; ICU: Intensive care unit; HLOS: Hospital length of stay; D-L: Dersimonian and Laird; Cl: Confidence interval; I $^{2}$ : Measure of heterogeneity; COVID-19: Coronavirus disease-19; PRISMA: Preferred reporting items for systematic reviews and meta-analyses; GLM: Generalized linear model; DM: Diabetes; HTN: Hypertension; AMR: Antimicrobial resistance

\section{Supplementary Information}

The online version contains supplementary material available at https://doi. org/10.1186/s12884-021-03685-w.

Additional file 1: Supplementary Table 1. Methodological quality assessment for studies included in the meta-analysis. Supplementary Table 2. Assessment of small studies effects (publication bias). Supplementary Table 3. Associations of maternal characteristics and treatments with outcomes in case reports.

Additional file 2: Supplementary Figure 1. Funnel plots for proportions of intensive care unit admission and preterm birth outcomes.

Additional file 3: Supplementary Figure 2. Pattern of steroids and hydroxychloroquine use among pregnant women infected with SARSCov-2 in case series studies.

\section{Acknowledgements}

We appreciate the efforts of all the researchers whose articles were included in this study.

\section{Authors' contributions}

PD designed and conceptualized study; involved in acquisition of data; drafted the manuscript; PD, BT reviewed all articles for their eligibility, conducted quality assessment of each study independently, extracted data and verified the underlying data; BT and AD performed meta-analysis and prepared results, figures, and tables; SR and $A D$ assisted in design, presentation, and interpretation of results; CM, CB, SM, SC, and NM interpreted the data; revised the manuscript for intellectual content; All authors have all authors have read and approved the manuscript, substantially contributed to in the interpretation of results, and edited manuscript.

Funding

No financial support was received for this study.

Availability of data and materials

The data that support the findings of this study are available with corresponding authors upon reasonable request.

\section{Declarations}

Ethics approval and consent to participate

Not applicable.

Consent for publication

Not applicable.

\section{Competing interests}

All authors declare that they have no competing interests.

\section{Author details}

1 Department of Obstetrics and Gynecology, Paul L. Foster School of Medicine, Texas Tech University Health Sciences Center El Paso, El Paso, TX, USA. ${ }^{2}$ Division of Biostatistics \& Epidemiology, Department of Molecular and Translational Medicine, Paul L. Foster School of Medicine, Texas Tech University Health Sciences Center El Paso, El Paso, TX, USA. ${ }^{3}$ School of Pharmacy, the University of Texas at El Paso, El Paso, TX, USA. ${ }^{4}$ Department of Pediatrics, Paul L. Foster School of Medicine, Texas Tech University Health Sciences Center El Paso, El Paso, TX, USA. ${ }^{5}$ Biostatistics and Epidemiology Consulting Lab, Texas Tech University Health Sciences Center El Paso, El Paso, TX, USA.

Received: 8 November 2020 Accepted: 28 February 2021

Published online: 24 March 2021

References

1. WHO Health Emergency Dashboard WHO (COVID-19) Homepage. https:// covid19.who.int/.

2. Hu Y, Sun J, Dai Z, Deng H, Li X, Huang Q, Wu Y, Sun L, Xu Y. Prevalence and severity of corona virus disease 2019 (COVID-19): a systematic review and meta-analysis. J Clin Virol. 2020;127:104371. 
3. Rabaan AA, Al-Ahmed SH, Haque S, Sah R, Tiwari R, Malik YS, Dhama K, Yatoo MI, Bonilla-Aldana DK, Rodriguez-Morales AJ. SARS-CoV-2, SARS-CoV, and MERS-COV: a comparative overview. Infez Med. 2020;28(2):174-84.

4. Racicot $K$, Mor G. Risks associated with viral infections during pregnancy. J Clin Invest. 2017;127(5):1591-9.

5. Khalil A, Kalafat E, Benlioglu C, O'Brien P, Morris E, Draycott T, Thangaratinam S, Le Doare K, Heath P, Ladhani S, et al. SARS-CoV-2 infection in pregnancy: a systematic review and meta-analysis of clinical features and pregnancy outcomes. EClinicalMedicine. 2020;25:100446.

6. Ellington S, Strid P, Tong VT, Woodworth K, Galang RR, Zambrano LD, Nahabedian J, Anderson K, Gilboa SM. Characteristics of women of reproductive age with laboratory-confirmed SARS-CoV-2 infection by pregnancy status - United States, January 22-June 7, 2020. MMWR Morb Mortal Wkly Rep. 2020;69(25):769-75.

7. Li L, Wang X, Wang R, Hu Y, Jiang S, Lu X. Antiviral agent therapy optimization in special populations of COVID-19 patients. Drug Des Devel Ther. 2020;14:3001-13.

8. Anderson J, Schauer J, Bryant S, Graves CR. The use of convalescent plasma therapy and remdesivir in the successful management of a critically ill obstetric patient with novel coronavirus 2019 infection: a case report. Case Rep Womens Health. 2020;27:e00221.

9. Williamson BN, Feldmann F, Schwarz B, et al. Clinical benefit of remdesivir in rhesus macaques infected with SARS-CoV-2. Nature. 2020;585:273-6.

10. RECOVERY Collaborative Group, Horby P, Lim WS, Emberson JR, Mafham M, Bell JL, Linsell L, Staplin N, Brightling C, Ustianowski A, Elmahi E, Prudon B, Green C, Felton T, Chadwick D, Rege K, Fegan C, Chappell LC, Faust SN, Jaki T, Jeffery K, Montgomery A, Rowan K, Juszczak E, Baillie JK, Haynes R, Landray MJ. Dexamethasone in Hospitalized Patients with Covid-19. N Engl J Med. 2021;384(8):693-704. https://doi.org/10.1056/NEJMoa2021436.

11. Prescott HC, Rice TW. Corticosteroids in COVID-19 ARDS: evidence and Hope during the pandemic. JAMA. 2020;324(13):1292-95. https://doi.org/1 0.1001/jama.2020.16747.

12. Thomson $\mathrm{K}$, Nachlis $\mathrm{H}$. Emergency use authorizations during the COVID-19 pandemic: lessons from Hydroxychloroquine for vaccine authorization and approval. JAMA. 2020;324(13):1282-3.

13. RECOVERY Collaborative Group, Horby P, Mafham M, Linsell L, Bell JL, Staplin N, Emberson JR, Wiselka M, Ustianowski A, Elmahi E, Prudon B, Whitehouse T, Felton T, Williams J, Faccenda J, Underwood J, Baillie JK, Chappell LC, Faust SN, Jaki T, Jeffery K, Lim WS, Montgomery A, Rowan K, Tarning J, Watson JA, White NJ, Juszczak E, Haynes R, Landray MJ. Effect of Hydroxychloroquine in Hospitalized Patients with Covid-19. N Engl J Med. 2020;383(21):2030-40. https://doi.org/10.1056/NEJMoa2022926.

14. Vaughn VM, Gandhi T, Petty LA, Patel PK, Prescott HC, Malani AN, Ratz D, McLaughlin E, Chopra V, Flanders SA. Empiric Antibacterial Therapy and Community-onset Bacterial Co-infection in Patients Hospitalized with COVID-19: A Multi-Hospital Cohort Study. Clin Infect Dis. 2020;ciaa1239. https://doi.org/10.1093/cid/ciaa1239. Epub ahead of print.

15. Rico-Mesa JS, Rosas D, Ahmadian-Tehrani A, White A, Anderson AS, Chilton $\mathrm{R}$. The role of anticoagulation in COVID-19-induced hypercoagulability. Curr Cardiol Rep. 2020;22(7):53. https://doi.org/10.1007/s11886-020-01328-8.

16. Pawar AY, Hiray AP, Sonawane DD, Bhambar RS, Derle DV, Ahire YS. Convalescent plasma: a possible treatment protocol for COVID- 19 patients suffering from diabetes or underlying liver diseases. Diabetes Metab Syndr. 2020;14(4):665-9.

17. Kumar A, Kubota Y, Chernov M, Kasuya H. Potential role of zinc supplementation in prophylaxis and treatment of COVID-19. Med Hypotheses. 2020;144:109848.

18. Mei Y, Luo D, Wei S, Liao X, Pan Y, Yang X, Lin Y. Obstetric Management of COVID-19 in Pregnant Women. Front Microbiol. 2020;11:1186. https://doi. org/10.3389/fmicb.2020.01186.

19. Dubey P, Reddy SY, Manuel S, Dwivedi AK. Maternal and neonatal characteristics and outcomes among COVID-19 infected women: An updated systematic review and meta-analysis. Eur J Obstet Gynecol Reprod Biol. 2020;252:490-501

20. Liberati A, Altman DG, Tetzlaff J, Mulrow C, Gøtzsche PC, loannidis JPA, Clarke M, Devereaux PJ, Kleijnen J, Moher D. The PRISMA statement for reporting systematic reviews and meta-analyses of studies that evaluate healthcare interventions: explanation and elaboration. BMJ. 2009;339:b2700.

21. Stroup DF, Berlin JA, Morton SC, Olkin I, Williamson GD, Rennie D, Moher D, Becker BJ, Sipe TA, Thacker SB. Meta-analysis of observational studies in epidemiology: a proposal for reporting. Meta-analysis of observational studies in epidemiology (MOOSE) group. JAMA. 2000;283(15):2008-12.

22. Dwivedi AK, Shukla R. Evidence-based statistical analysis and methods in biomedical research (SAMBR) checklists according to design features. Cancer Rep (Hoboken). 2020;3(4):e1211.

23. Chen H, Guo J, Wang C, Luo F, Yu X, Zhang W, Li J, Zhao D, Xu D, Gong Q, et al. Clinical characteristics and intrauterine vertical transmission potential of COVID-19 infection in nine pregnant women: a retrospective review of medical records. Lancet. 2020;395(10226):809-15.

24. Liu D, Li L, Wu X, Zheng D, Wang J, Yang L, Zheng C. Pregnancy and perinatal outcomes of women with coronavirus disease (COVID-19) pneumonia: a preliminary analysis. AJR Am J Roentgenol. 2020;215(1):127-32.

25. Li N, Han L, Peng M, Lv Y, Ouyang Y, Liu K, Yue L, Li Q, Sun G, Chen L, Yang $L$. Maternal and neonatal outcomes of pregnant women with coronavirus disease 2019 (COVID-19) pneumonia: A case-control study. Clin Infect Dis. 2020;71(16):2035-41. https://doi.org/10.1093/cid/ciaa352.

26. Liu W, Wang J, Li W, Zhou Z, Liu S, Rong Z. Clinical characteristics of 19 neonates born to mothers with COVID-19. Front Med. 2020;14(2):193-8.

27. Hantoushzadeh S, Shamshirsaz AA, Aleyasin A, Seferovic MD, Aski SK, Arian SE, Pooransari P, Ghotbizadeh F, Aalipour S, Soleimani Z, et al. Maternal death due to COVID-19. Am J Obstet Gynecol. 2020;223(1):109 e101-16.

28. London V, McLaren R Jr, Atallah F, Cepeda C, McCalla S, Fisher N, Stein JL, Haberman S, Minkoff $\mathrm{H}$. The relationship between status at presentation and outcomes among pregnant women with COVID-19. Am J Perinatol. 2020;37(10):991-4.

29. Chen Y, Bai J. Maternal and infant outcomes of full-term pregnancy combined with COVID-2019 in Wuhan, China: retrospective case series. Arch Gynecol Obstet. 2020;302(3):545-51.

30. Savasi VM, Parisi F, Patane L, Ferrazzi E, Frigerio L, Pellegrino A, Spinillo A, Tateo S, Ottoboni M, Veronese P, et al. Clinical findings and disease severity in hospitalized pregnant women with coronavirus disease 2019 (COVID-19). Obstet Gynecol. 2020;136(2):252-8.

31. Zeng Y, Lin L, Yan Q, Wei W, Xiang Yang B, Huang R, He F, Chen D. Update on clinical outcomes of women with COVID-19 during pregnancy. Int J Gynaecol Obstet. 2020;150(2):264-6.

32. Lokken EM, Walker CL, Delaney S, Kachikis A, Kretzer NM, Erickson A, Resnick R, Vanderhoeven J, Hwang JK, Barnhart N, Rah J, McCartney SA, Ma KK, Huebner EM, Thomas C, Sheng JS, Paek BW, Retzlaff K, Kline CR, Munson J, Blain M, LaCourse SM, Deutsch G, Adams Waldorf KM. Clinical characteristics of 46 pregnant women with a severe acute respiratory syndrome coronavirus 2 infection in Washington State. Am J Obstet Gynecol. 2020; 223(6):911.e1-911.e14. https://doi.org/10.1016/j.ajog.2020.05.031.

33. San-Juan R, Barbero P, Fernández-Ruiz M, López-Medrano F, Lizasoáin M, Hernández-Jiménez P, Silva JT, Ruiz-Ruigómez M, Corbella L, RodríguezGoncer I, Folgueira MD, Lalueza A, Batllori E, Mejía I, Forcén L, Lumbreras C, García-Burguillo A, Galindo A, Aguado JM. Incidence and clinical profiles of COVID-19 pneumonia in pregnant women: A single-centre cohort study from Spain. EClinicalMedicine. 2020;23:100407. https://doi.org/10.1016/j. eclinm.2020.100407.

34. Zhang L, Dong L, Ming L, Wei M, Li J, Hu R, Yang J. Severe acute respiratory syndrome coronavirus 2(SARS-CoV-2) infection during late pregnancy: a report of 18 patients from Wuhan, China. BMC Pregnancy Childbirth. 2020; 20(1):394.

35. Sentilhes L, De Marcillac F, Jouffrieau C, Kuhn P, Thuet V, Hansmann Y, Ruch Y, Fafi-Kremer S, Deruelle P. Coronavirus disease 2019 in pregnancy was associated with maternal morbidity and preterm birth. Am J Obstet Gynecol. 2020;223(6):914.e1-914.e15. https://doi.org/10.1016/j.ajog.2020.06.022.

36. Blitz MJ, Rochelson B, Minkoff H, Meirowitz N, Prasannan L, London V, Rafael TJ, Chakravarthy S, Bracero LA, Wasden SW, et al. Maternal mortality among women with coronavirus disease 2019 admitted to the intensive care unit. Am J Obstet Gynecol. 2020;223(4):595-9 e595.

37. Prabhu M, Cagino K, Matthews KC, Friedlander RL, Glynn SM, Kubiak JM, Yang YJ, Zhao Z, Baergen RN, DiPace Jl, et al. Pregnancy and postpartum outcomes in a universally tested population for SARS-CoV-2 in New York City: a prospective cohort study. BJOG. 2020;127(12):1548-56.

38. Vivanti AJ, Mattern J, Vauloup-Fellous C, Jani J, Rigonnot L, El Hachem L, Le Gouez A, Desconclois C, Ben M'Barek I, Sibiude J, et al. Retrospective description of pregnant women infected with severe acute respiratory syndrome coronavirus 2, France. Emerg Infect Dis. 2020;26(9):2069-76. 
39. Marín Gabriel MA, Cuadrado I, Álvarez Fernández B, González Carrasco E, Alonso Díaz C, Llana Martín I, Sánchez L, Olivas C, de Las Heras S, Criado E; Neo-COVID-19 Research Group. Multicentre Spanish study found no incidences of viral transmission in infants born to mothers with COVID-19. Acta Paediatr. 2020;109(11):2302-8. https://doi.org/10.1111/apa.15474.

40. Sahin D, Tanacan A, Erol SA, Anuk AT, Eyi EGY, Ozgu-Erdinc AS, Yucel A, Keskin HL, Tayman C, Unlu S, Kirca F, Dinc B, San I, Parpucu ÜM, Surel AA, Moraloglu OT. A pandemic center's experience of managing pregnant women with COVID-19 infection in Turkey: A prospective cohort study. Int J Gynaecol Obstet. 2020;151(1):74-82. https://doi.org/10.1002/ijgo.13318

41. Barbero P, Muguerza L, Herraiz I, Garcia Burguillo A, San Juan R, Forcen L, Mejia I, Batllori E, Montanez MD, Vallejo P, et al. SARS-CoV-2 in pregnancy: characteristics and outcomes of hospitalized and non-hospitalized women due to COVID-19. J Matern Fetal Neonatal Med. 2020:1-7.

42. Emeruwa UN, Spiegelman J, Ona S, Kahe K, Miller RS, Fuchs KM, Aubey JJ, Booker W, D'Alton ME, Friedman AM, Aziz A, Sutton D, Purisch SE, Goffman D, Melamed A, Gyamfi-Bannerman C. Influence of Race and Ethnicity on Severe Acute Respiratory Syndrome Coronavirus 2 (SARS-CoV-2) Infection Rates and Clinical Outcomes in Pregnancy. Obstet Gynecol. 2020;136(5): 1040-43. https://doi.org/10.1097/AOG.0000000000004088.

43. Xu S, Shao F, Bao B, Ma X, Xu Z, You J, Zhao P, Liu Y, Ng M, Cui H, et al. Clinical Manifestation and Neonatal Outcomes of Pregnant Patients With Coronavirus Disease 2019 Pneumonia in Wuhan, China. Open Forum Infect Dis. 2020;7(7):ofaa283.

44. Oncel MY, Akın IM, Kanburoglu MK, Tayman C, Coskun S, Narter F, Er I, Oncan TG, Memisoglu A, Cetinkaya M, Oguz D, Erdeve O, Koc E; Neo-Covid Study Group. A multicenter study on epidemiological and clinical characteristics of 125 newborns born to women infected with COVID-19 by Turkish Neonatal Society. Eur J Pediatr. 2021;180(3):733-42. https://doi. org/10.1007/s00431-020-03767-5.

45. Nayak AH, Kapote DS, Fonseca M, Chavan N, Mayekar R, Sarmalkar M, Bawa A. Impact of the coronavirus infection in pregnancy: a preliminary study of 141 patients. J Obstet Gynaecol India. 2020;70(4):256-61.

46. Wang X, Zhou Z, Zhang J, Zhu F, Tang Y, Shen X. A case of 2019 novel coronavirus in a pregnant woman with preterm delivery. Clin Infect Dis. 2020;71(15):844-6.

47. Li Y, Zhao R, Zheng S, Chen X, Wang J, Sheng X, Zhou J, Cai H, Fang Q, Yu $F$, et al. Lack of vertical transmission of severe acute respiratory syndrome coronavirus 2, China. Emerg Infect Dis. 2020;26(6):1335-6.

48. Wang S, Guo L, Chen L, Liu W, Cao Y, Zhang J, Feng L. A case report of neonatal 2019 coronavirus disease in China. Clin Infect Dis. 2020;71(15):853-7.

49. Fan C, Lei D, Fang C, Li C, Wang M, Liu Y, Bao Y, Sun Y, Huang J, Guo Y, Yu Y, Wang S. Perinatal Transmission of 2019 Coronavirus Disease-Associated Severe Acute Respiratory Syndrome Coronavirus 2: Should We Worry? Clin Infect Dis. 2021;72(5):862-4. https://doi.org/10.1093/cid/ciaa226.

50. Chen S, Liao E, Cao D, Gao Y, Sun G, Shao Y. Clinical analysis of pregnant women with 2019 novel coronavirus pneumonia. J Med Virol. 2020;92(9): 1556-61. https://doi.org/10.1002/jmv.25789.

51. Lee DH, Lee J, Kim E, Woo K, Park HY, An J. Emergency cesarean section performed in a patient with confirmed severe acute respiratory syndrome Coronavirus-2 -a case report. Korean J Anesthesiol. 2020;73(4):347-51.

52. Kalafat E, Yaprak E, Cinar G, Varli B, Ozisik S, Uzun C, Azap A, Koc A. Lung ultrasound and computed tomographic findings in pregnant woman with COVID-19. Ultrasound Obstet Gynecol. 2020;55(6):835-7.

53. Gidlof S, Savchenko J, Brune T, Josefsson H. COVID-19 in pregnancy with comorbidities: more liberal testing strategy is needed. Acta Obstet Gynecol Scand. 2020;99(7):948-9.

54. Khan S, Peng L, Siddique R, Nabi G, Nawsherwan, Xue M, Liu J, Han G. Impact of COVID-19 infection on pregnancy outcomes and the risk of maternal-to-neonatal intrapartum transmission of COVID-19 during natural birth. Infect Control Hosp Epidemiol. 2020;41 (6):748-50.

55. Iqbal SN, Overcash R, Mokhtari N, Saeed H, Gold S, Auguste T, Mirza MU, Ruiz ME, Chahine JJ, Waga M, et al. An uncomplicated delivery in a patient with Covid-19 in the United States. N Engl J Med. 2020;382(16):e34.

56. Alzamora MC, Paredes T, Caceres D, Webb CM, Valdez LM, La Rosa M. Severe COVID-19 during pregnancy and possible vertical transmission. Am J Perinatol. 2020;37(8):861-5.

57. Peng Z, Wang J, Mo Y, Duan W, Xiang G, Yi M, Bao L, Shi Y. Unlikely SARSCoV-2 vertical transmission from mother to child: a case report. J Infect Public Health. 2020;13(5):818-20.
58. González Romero D, Ocampo Pérez J, González Bautista L, Santana-Cabrera L. Pronóstico perinatal y de la paciente embarazada con infección por COVID-19 [Pregnancy and perinatal outcome of a woman with COVID-19 infection]. Rev Clin Esp. 2020;220(8):533-4. Spanish. https://doi.org/10.1016/j. rce.2020.04.006

59. Lu D, Sang L, Du S, Li T, Chang Y, Yang XA. Asymptomatic COVID-19 infection in late pregnancy indicated no vertical transmission. J Med Virol. 2020;92(9):1660-64. https://doi.org/10.1002/jmv.25927.

60. Browne PC, Linfert JB, Perez-Jorge E. Successful treatment of preterm labor in association with acute COVID-19 infection. Am J Perinatol. 2020;37(8): 866-8.

61. Blauvelt CA, Chiu C, Donovan AL, Prahl M, Shimotake TK, George RB, Schwartz BS, Faroogi NA, Ali SS, Cassidy A, et al. Acute respiratory distress syndrome in a preterm pregnant patient with coronavirus disease 2019 (COVID-19). Obstet Gynecol. 2020;136(1):46-51.

62. Silverstein JS, Limaye MA, Brubaker SG, Roman AS, Bautista J, Chervenak J, Ratner AJ, Sommer PM, Roselli NM, Gibson CD, et al. Acute respiratory Decompensation requiring intubation in pregnant women with SARS-CoV-2 (COVID-19). AJP Rep. 2020;10(2):e169-75.

63. AlZaghal LA, AlZaghal N, Alomari SO, Obeidat N, Obeidat B, Hayajneh WA. Multidisciplinary team management and cesarean delivery for a Jordanian woman infected with SARS-COV-2: a case report. Case Rep Womens Health. 2020;27:e00212.

64. Li J, Wang Y, Zeng Y, Song T, Pan X, Jia M, He F, Hou L, Li B, He S, et al. Critically ill pregnant patient with COVID-19 and neonatal death within two hours of birth. Int J Gynaecol Obstet. 2020;150(1):126-8.

65. Hong L, Smith N, Keerthy M, Lee-Griffith M, Garcia R, Shaman M, Goyert G. Severe COVID-19 infection in pregnancy requiring intubation without preterm delivery: a case report. Case Rep Womens Health. 2020;27:e00217.

66. Taghizadieh A, Mikaeili H, Ahmadi M, Valizadeh H. Acute kidney injury in pregnant women following SARS-CoV-2 infection: a case report from Iran. Respir Med Case Rep. 2020;30:101090.

67. Cooke WR, Billett A, Gleeson S, Jacques A, Place K, Siddall J, Walden A, Soulsby K. SARS-CoV-2 infection in very preterm pregnancy: experiences from two cases. Eur J Obstet Gynecol Reprod Biol. 2020;250:259-60.

68. Mehta H, Ivanovic S, Cronin A, VanBrunt L, Mistry N, Miller R, Yodice P, Rezai $F$. Novel coronavirus-related acute respiratory distress syndrome in a patient with twin pregnancy: a case report. Case Rep Womens Health. 2020;27: e00220.

69. Yu Y, Fan C, Bian J, YinShen. Severe COVID-19 in a pregnant patient admitted to hospital in Wuhan. Int J Gynaecol Obstet. 2020;150(2):262-3.

70. Fontanella F, Hannes S, Keating N, Martyn F, Browne I, Briet J, McAuliffe FM, Baalman JH. COVID-19 infection during the third trimester of pregnancy: current clinical dilemmas. Eur J Obstet Gynecol Reprod Biol. 2020;251:268-71.

71. Grimminck K, Santegoets LAM, Siemens FC, Fraaij PLA, Reiss IKM, Schoenmakers S. No evidence of vertical transmission of SARS-CoV-2 after induction of labour in an immune-suppressed SARS-CoV-2-positive patient. BMJ Case Rep. 2020;13(6):e235581. https://doi.org/10.1136/bcr-2020-235581.

72. Naqvi M, Zakowski P, Glucksman L, Smithson S, Burwick RM. Tocilizumab and Remdesivir in a Pregnant Patient With Coronavirus Disease 2019 (COVID-19). Obstet Gynecol. 2020;136(5):1025-29. https://doi.org/10.1097/A OG.0000000000004050.

73. Zheng T, Guo J, He W, Wang H, Yu H, Ye H. Coronavirus disease 2019 (COVID-19) in pregnancy: 2 case reports on maternal and neonatal outcomes in Yichang city, Hubei Province, China. Medicine (Baltimore). 2020;99(29):e21334.

74. Douedi S, Albayati A, Alfraji N, Mazahir U, Costanzo E. Successful maternal and fetal outcomes in COVID-19 pregnant women: An institutional approach. Am J Case Rep. 2020;21:e925513.

75. Marzollo R, Aversa S, Prefumo F, Saccani B, Perez CR, Sartori E, Motta M. Possible coronavirus disease 2019 pandemic and pregnancy: vertical transmission is not excluded. Pediatr Infect Dis J. 2020;39(9):e261-2.

76. Reis H, Boldrini NAT, Caldas JVJ, Paz A, Ferrugini CLP, Miranda AE. Severe coronavirus infection in pregnancy: challenging cases report. Rev Inst Med Trop Sao Paulo. 2020;62:e49.

77. Oliva M, Hsu K, Alsamarai S, Chavez V, Ferrara L. Clinical improvement of severe COVID-19 pneumonia in a pregnant patient after caesarean delivery. BMJ Case Rep. 2020;13(7):e236290. https://doi.org/10.1136/bcr-2020-236290.

78. Richtmann R, Torloni MR, Oyamada Otani AR, Levi JE, Crema Tobara M, de Almeida SC, Dias L, Miglioli-Galvao L, Martins Silva P, Macoto Kondo M. Fetal 
deaths in pregnancies with SARS-CoV-2 infection in Brazil: a case series. Case Rep Womens Health. 2020;27:e00243.

79. Kolkova Z, Bjurstrom MF, Lansberg JK, Svedas E, Hamer MA, Hansson SR, Herbst A, Zaigham M. Obstetric and intensive-care strategies in a high-risk pregnancy with critical respiratory failure due to COVID-19: a case report. Case Rep Womens Health. 2020;27:e00240.

80. Soleimani Z, Soleimani A. ADRS due to COVID-19 in midterm pregnancy: successful management with plasma transfusion and corticosteroids. J Matern Fetal Neonatal Med. 2020:1-4.

81. Easterlin MC, De Beritto T, Yeh AM, Wertheimer FB, Ramanathan R Extremely preterm infant born to a mother with severe COVID-19 pneumonia. J Investig Med High Impact Case Rep. 2020;8: 2324709620946621.

82. Chong J, Ahmed S, Hill K. Acute respiratory distress syndrome in a pregnant patient with COVID-19 improved after delivery: a case report and brief review. Respir Med Case Rep. 2020;31:101171.

83. Ahmed I, Eltaweel N, Antoun L, Rehal A. Severe pre-eclampsia complicated by acute fatty liver disease of pregnancy, HELLP syndrome and acute kidney injury following SARS-CoV-2 infection. BMJ Case Rep. 2020;13(8):e237521. https://doi.org/10.1136/bcr-2020-237521.

84. Chhabra A, Rao TN, Kumar M, Singh Y, Subramaniam R. Anaesthetic management of a COVID-19 parturient for caesarean section - case report and lessons learnt. Indian J Anaesth. 2020;64(Suppl 2):S141-3.

85. Figueiredo R, Falcão V, Pinto MJ, Ramalho C. Peripheral facial paralysis as presenting symptom of COVID-19 in a pregnant woman. BMJ Case Rep. 2020;13(8):e237146. https://doi.org/10.1136/bcr-2020-237146.

86. Federici L, Picone O, Dreyfuss D, Sibiude J. Successful continuation of pregnancy in a patient with COVID-19-related ARDS. BMJ Case Rep. 2020; 13(8):e237511. https://doi.org/10.1136/bcr-2020-237511.

87. Peng J, Li R, Yin H, Tang F, Xie H, Li M, Zhao Y. A case report of a pregnant woman infected with coronavirus disease 2019 pneumonia. Medicine (Baltimore). 2020:99(30):e21335.

88. Boehmer TK, DeVies J, Caruso E, van Santen KL, Tang S, Black CL, Hartnett KP, Kite-Powell A, Dietz S, Lozier M, et al. Changing age distribution of the COVID-19 pandemic - United States, may-august 2020. MMWR Morb Mortal Wkly Rep. 2020;69(39):1404-9.

89. Breslin N, Baptiste C, Miller R, Fuchs K, Goffman D, Gyamfi-Bannerman C, D'Alton M. Coronavirus disease 2019 in pregnancy: early lessons. Am J Obstet Gynecol MFM. 2020;2(2):100111.

90. Delahoy MJ, Whitaker M, O'Halloran A, Chai SJ, Kirley PD, Alden N, Kawasaki B, Meek J, Yousey-Hindes K, Anderson EJ, et al. Characteristics and maternal and birth outcomes of hospitalized pregnant women with laboratory-confirmed COVID-19 - COVID-NET, 13 states, march 1-august 22, 2020. MMWR Morb Mortal Wkly Rep. 2020;69(38):1347-54.

91. Allotey J, Stallings E, Bonet M, Yap M, Chatterjee S, Kew T, Debenham L, Llavall AC, Dixit A, Zhou D, et al. Clinical manifestations, risk factors, and maternal and perinatal outcomes of coronavirus disease 2019 in pregnancy: living systematic review and meta-analysis. BMJ. 2020;370: m3320.

92. Kim AY, Gandhi RT. Coronavirus disease 2019 (COVID-19): Management in hospitalized adults. Section Editor: Martin S. Hirsch. Deputy Editor: Allyson Bloom. UpToDate; Literature review current through: Feb 2021. This topic last updated: Feb 26, 2021. (https://www.uptodate.com/contents/corona virus-disease-2019-covid-19-management-in-hospitalized-adults).

93. Beovic B, Dousak M, Ferreira-Coimbra J, Nadrah K, Rubulotta F, Belliato M, Berger-Estilita J, Ayoade F, Rello J, Erdem H. Antibiotic use in patients with COVID-19: a 'snapshot' infectious diseases international research initiative (ID-IRI) survey. J Antimicrob Chemother. 2020;75(11): 3386-90.

94. Getahun H, Smith I, Trivedi K, Paulin S, Balkhy HH. Tackling antimicrobial resistance in the COVID-19 pandemic. Bull World Health Organ. 2020;98(7): 442-442A.

95. Bowes WA. The role of antibiotics in the prevention of preterm birth. F1000 Med Rep. 2009;1:22. https://doi.org/10.3410/M1-22.

96. Clancy CJ, Buehrle DJ, Nguyen MH. PRO: The COVID-19 pandemic will result in increased antimicrobial resistance rates. JAC-Antimicrobial Resistance. 2020;2(3):dlaa049. https://doi.org/10.1093/jacamr/dlaa049.

97. Thng ZX, De Smet MD, Lee CS, Gupta V, Smith JR, McCluskey PJ, Thorne JE, Kempen JH, Zierhut M, Nguyen QD, Pavesio C, Agrawal R. COVID-19 and immunosuppression: a review of current clinical experiences and implications for ophthalmology patients taking immunosuppressive drugs. Br J Ophthalmol. 2021;105(3):306-10. https://doi.org/10.1136/bjophthalmol-2 020-316586.

98. Beigel JH, Tomashek KM, Dodd LE, Mehta AK, Zingman BS, Kalil AC, Hohmann E, Chu HY, Luetkemeyer A, Kline S, Lopez de Castilla D, Finberg RW, Dierberg K, Tapson V, Hsieh L, Patterson TF, Paredes R, Sweeney DA, Short WR, Touloumi G, Lye DC, Ohmagari N, Oh MD, Ruiz-Palacios GM, Benfield T, Fätkenheuer G, Kortepeter MG, Atmar RL, Creech CB, Lundgren J, Babiker AG, Pett S, Neaton JD, Burgess TH, Bonnett T, Green M, Makowski M, Osinusi A, Nayak S, Lane HC; ACTT-1 Study Group Members. Remdesivir for the Treatment of Covid-19 - Final Report. N Engl J Med. 2020;383(19):181326. https://doi.org/10.1056/NEJMoa2007764.

99. Nielsen Jeschke K, Bonnesen B, Hansen EF, Jensen JS, Lapperre TS, Weinreich UM, Hilberg O. Guideline for the management of COVID-19 patients during hospital admission in a non-intensive care setting. Eur Clin Respir J. 2020;7(1):1761677.

100. Barbateskovic M, Schjørring OL, Russo Krauss S, Jakobsen JC, Meyhoff CS, Dahl RM, Rasmussen BS, Perner A, Wetterslev J. Higher versus lower fraction of inspired oxygen or targets of arterial oxygenation for adults admitted to the intensive care unit. Cochrane Database Syst Rev. 2019;2019(11): CD012631. https://doi.org/10.1002/14651858.CD012631.

101. Lin KJ, Schneeweiss S, Tesfaye H, D'Andrea E, Liu J, Lii J, Murphy SN, Gagne JJ. Pharmacotherapy for hospitalized patients with COVID-19: treatment patterns by disease severity. Drugs. 2020;80(18):1961-72.

102. Geleris J, Sun Y, Platt J, Zucker J, Baldwin M, Hripcsak G, Labella A, Manson DK, Kubin C, Barr RG, Sobieszczyk ME, Schluger NW. Observational Study of Hydroxychloroquine in Hospitalized Patients with Covid-19. N Engl J Med. 2020;382(25):2411-18.

103. Mayor-Ibarguren A, Busca-Arenzana C, Robles-Marhuenda Á. A Hypothesis for the Possible Role of Zinc in the Immunological Pathways Related to COVID-19 Infection. Front Immunol. 2020;10(11):1736.

104. Stefanovic V. COVID-19 infection during pregnancy: fetus as a patient deserves more attention. J Perinat Med. 2020:48(5):438-40.

\section{Publisher's Note}

Springer Nature remains neutral with regard to jurisdictional claims in published maps and institutional affiliations.

\section{Ready to submit your research? Choose BMC and benefit from:}

- fast, convenient online submission

- thorough peer review by experienced researchers in your field

- rapid publication on acceptance

- support for research data, including large and complex data types

- gold Open Access which fosters wider collaboration and increased citations

- maximum visibility for your research: over $100 \mathrm{M}$ website views per year

At BMC, research is always in progress.

Learn more biomedcentral.com/submissions 